\title{
POWER DIAGRAMS AND INTERACTION PROCESSES FOR UNIONS OF DISCS
}

\author{
JESPER MØLLER, ${ }^{*}$ Aalborg University \\ KATEŘINA HELISOVÁ, ${ }^{* *}$ Charles University in Prague
}

\begin{abstract}
We study a flexible class of finite-disc process models with interaction between the discs. We let $\mathcal{U}$ denote the random set given by the union of discs, and use for the disc process an exponential family density with the canonical sufficient statistic depending only on geometric properties of $\mathcal{U}$ such as the area, perimeter, Euler-Poincaré characteristic, and the number of holes. This includes the quermass-interaction process and the continuum random-cluster model as special cases. Viewing our model as a connected component Markov point process, and thereby establishing local and spatial Markov properties, becomes useful for handling the problem of edge effects when only $u$ is observed within a bounded observation window. The power tessellation and its dual graph become major tools when establishing inclusion-exclusion formulae, formulae for computing geometric characteristics of $u$, and stability properties of the underlying disc process density. Algorithms for constructing the power tessellation of $u$ and for simulating the disc process are discussed, and the software is made public available.

Keywords: Area-interaction process; Boolean model; disc process; exponential family; germ-grain model; local computations; local stability; Markov properties; inclusionexclusion formulae; interaction; point process; power tessellation; simulation; quermassinteraction process; random closed set; Ruelle stability
\end{abstract}

2000 Mathematics Subject Classification: Primary 60D05; 60G55; 60K35; 62M30

Secondary $68 \mathrm{U} 20$

\section{Introduction}

This paper concerns probabilistic results of statistical relevance for planar random set models given by a finite union of discs $\mathcal{U}=\mathcal{U}_{X}$, where $X$ denotes the corresponding finite process of discs. We distinguish between the case where we can observe the discs in $X$ and the random set case where only (or at most) $\mathcal{U}$ is observed. The latter case occurs frequently in applications and will be of main interest to us.

Our random closed set $\mathcal{U}$ is a particular example of a germ-grain model [17], with the grains being discs. It is well known that any random closed set whose realizations are locally finite unions of compact convex sets is a germ-grain model with convex and compact grains [43], [44]. However, in order to make statistical inference, we need to restrict attention to a much smaller class of models such as a random-disc process model, and indeed random-disc Boolean models play the main role in practice (see [41] and the references therein). The Boolean model is in an abstract setting given by a Poisson process of compact sets (the grains) with no interaction

Received 21 August 2007; revision received 1 February 2008.

* Postal address: Department of Mathematical Sciences, Aalborg University, Fredrik Bajers Vej 7G, DK-9220 Aalborg, Denmark. Email address: jm@math.aau.dk

** Postal address: Department of Probability and Mathematical Statistics, Charles University in Prague, Sokolovská 83, 18675 Praha 8, Czech Republic. Email address: helisova@karlin.mff.cuni.cz 
between the grains. Many authors (see, for example, [2], [8], [9], [16], [22], and [41]) have mentioned the need of developing flexible germ-grain models with interaction between the grains.

We study a particular class of models for interaction among the discs, specified by a point process density for $X$ with respect to a reference Poisson process of discs. The density is assumed to be of exponential family form with the canonical sufficient statistic $\boldsymbol{T}(X)=$ $\boldsymbol{T}(\mathcal{U})$ depending only on $X$ through $\mathcal{U}$, where $\boldsymbol{T}(\mathcal{U})$ is specified in terms of geometric characteristics for the connected components of $\mathcal{U}$, for example, the area $A(\mathcal{U})$, the perimeter $L(U)$, the number of holes $N_{\mathrm{h}}(U)$, and the number of connected components $N_{\mathrm{cc}}(U)$. Further geometric characteristics are specified in Section 4.1 in terms of the power tessellation (see, for example, [1]), which provides a subdivision of $\mathcal{U}$ (see Figure 1 in Section 3). An important special case of our models is the quermass-interaction process, first introduced in [22], where

$$
\boldsymbol{T}(\mathcal{U})=(A(\mathcal{U}), L(\mathcal{U}), \chi(\mathcal{U})) \text { and } \chi(\mathcal{U})=N_{\mathrm{cc}}(\mathcal{U})-N_{\mathrm{h}}(\mathcal{U})
$$

is the Euler-Poincaré characteristic (quermass integrals in $\mathbb{R}^{2}$ are linear combinations of $A, L$, and $\chi$ ). Another special case is the continuum random-cluster model [15], [23], [28], where $\boldsymbol{T}(\mathcal{U})=N_{\mathrm{cc}}(\mathcal{U})$. ing

We show that the power tessellation and its dual graph are extremely useful when establish-

(i) inclusion-exclusion formulae for $\boldsymbol{T}(U)$;

(ii) formulae for computing geometric characteristics of $u$;

(iii) Ruelle stability and local stability of the density of $X$, and thereby convergence properties of Markov chain Monte Carlo (MCMC) algorithms for simulating $X$.

Note that the use of power diagrams is confined to discs (or balls), while, for example, quermassinteraction processes for other types of grains have been studied in the literature. Among other things, we demonstrate that a main geometric result in [22] related to the issue of Ruelle stability is easily derived by means of the power tessellation and its dual graph. Furthermore, as explained in Section 4.5, it becomes useful to view our models as connected component Markov point processes [2], [4], [7], [30] in a similar way as the Markov connected component fields studied in [33]. In particular, we establish

(iv) local and spatial Markov properties of $X$, which become useful for handling the problem of edge effects when only $\mathcal{U}$ is observed within a bounded observation window.

The paper is organized as follows. In Section 2 we specify our notation and assumptions, and discuss a general position property of the discs in $X$. In Section 3 we define and study the power tessellation of a union of discs in general position. In the main section, Section 4, we study exponential family properties and the abovementioned issues (i)-(iv). Also, various examples of simulated realizations of our models are shown in Section 4. In Section 5 we discuss extensions of our work and some open problems. Finally, most algorithmic details are deferred to Appendix A.

A substantial part of this work has been the development of codes in C and R for constructing power tessellations and making simulations of our models. The codes are available at www.math.aau.dk/ jm/Codes.union.of.discs. 


\section{Preliminaries}

\subsection{Setup}

Throughout this paper, we use the following notation and make the following assumptions.

By a disc we mean more precisely a two-dimensional closed disc

$$
b=\left\{y \in \mathbb{R}^{2}:\|y-z\| \leq r\right\}
$$

with center $z \in \mathbb{R}^{2}$ and positive radius $r>0$, where $\|\cdot\|$ denotes the usual Euclidean distance. We identify $b$ with the point $x=(z, r)$ in $\mathbb{R}^{2} \times(0, \infty)$ and write $b=b(x)=b(z, r)$. Similarly, we identify point processes of discs $b_{i}=b\left(z_{i}, r_{i}\right)$ with point processes on $\mathbb{R}^{2} \times(0, \infty)$.

The reference point process will be a Poisson process $\Psi$ of discs; thus, the random set given by the union of discs in $\Psi$ is a Boolean model (see, for example, [27]). Specifically, $\Psi$ is assumed to be a Poisson point process on $\mathbb{R}^{2} \times(0, \infty)$ with an intensity measure of the form $\rho(z) \mathrm{d} z Q(\mathrm{~d} r)$, where $\mathrm{d} z$ is the Lebesgue measure on $\mathbb{R}^{2}$ and $Q$ is an arbitrary probability measure on $(0, \infty)$. In other words, the point process $\Phi$ of centers of discs given by $\Psi$ is a Poisson process with intensity function $\rho$ on $\mathbb{R}^{2}$, the radii of these discs are mutually independent and identically distributed with distribution $Q$, and $\Phi$ is independent of the radii. (An example of a simulation from such a process is shown in Figure 3(a)). The concrete specification of $\rho$ and $Q$ is not important for most results in this paper, but the specification is of course crucial for statistical inference (see [32]). Local integrability of $\rho$ is assumed to ensure that, with probability $1, \Phi \cap S$ is finite for any bounded region $S \subset \mathbb{R}^{2}$. Since we can view the radii as marks associated to the points given by the centers of the discs, we refer to $Q$ as the mark distribution. In the special case where $Q$ is degenerate at $R>0$, we can consider $R$ as a parameter and identify $\Psi$ with $\Phi$.

In the sequel $S$ denotes a given bounded planar region such that $\int_{S} \rho(z) \mathrm{d} z>0$. The object of primary interest is the random closed set

$$
u_{X}=\bigcup_{x \in X} b(x)
$$

where $X$ is a finite point process defined on $S \times(0, \infty)$. If $X=\varnothing$ is the empty configuration, we let $u_{X}=\varnothing$ be the empty set. Note that the centers of the discs are contained in $S$, but the discs may extend outside $S$. We assume that $X$ is absolutely continuous with respect to the reference Poisson process $\Psi$, and denote the density by $f(x)$ for finite configurations $x=\left\{x_{1}, \ldots, x_{n}\right\}$ with $x_{i}=\left(z_{i}, r_{i}\right) \in S \times(0, \infty)$ and $0 \leq n<\infty$ (if $n=0$ then $x$ is the empty configuration).

We focus on the case where the density is of the exponential family form, i.e.

$$
f_{\boldsymbol{\theta}}(x)=\frac{\exp \left(\boldsymbol{\theta} \cdot \boldsymbol{T}\left(\mathcal{U}_{x}\right)\right)}{c_{\boldsymbol{\theta}}}
$$

where $\boldsymbol{\theta}$ is a real parameter vector, ' $'$ ' denotes the usual inner product, $\boldsymbol{T}(\boldsymbol{U})$ is a statistic of the same dimension as $\boldsymbol{\theta}$, and $c_{\boldsymbol{\theta}}$ is a normalizing constant depending on $\boldsymbol{\theta}$ (and of course also on $(\boldsymbol{T}, \rho, Q)$ ). Note that $f_{\boldsymbol{\theta}}(x)>0$ for all $x$. Further details on the choice of $\boldsymbol{T}$ and the parameter space for $\boldsymbol{\theta}$ are given in Section 4. Note that (1) is also the density of the random set $U_{X}$ with 
respect to the reference Boolean model and that

$$
\begin{aligned}
c_{\boldsymbol{\theta}}= & \exp \left(-\int_{S} \rho(z) \mathrm{d} z\right) \\
\times & \left(\exp (\boldsymbol{\theta} \cdot \boldsymbol{T}(\varnothing))+\sum_{n=1}^{\infty} \frac{1}{n !} \int_{S} \int_{0}^{\infty} \cdots \int_{S} \int_{0}^{\infty} \exp \left(\boldsymbol { \theta } \cdot \boldsymbol { T } \left(\mathcal{U}_{\left.\left.\left\{\left(z_{1}, r_{1}\right), \ldots,\left(z_{n}, r_{n}\right)\right\}\right)\right)}\right.\right.\right. \\
& \left.\times \prod_{1}^{n} \rho\left(z_{i}\right) \mathrm{d} z_{1} Q\left(\mathrm{~d} r_{1}\right) \cdots \mathrm{d} z_{n} Q\left(\mathrm{~d} r_{n}\right)\right)
\end{aligned}
$$

is in general not expressible in closed form (unless $\boldsymbol{\theta}=\mathbf{0}$ ).

As noted in Section 1, a quermass-interaction process is obtained by taking $\boldsymbol{T}(\mathcal{U})=$ $(A(\mathcal{U}), L(\mathcal{U}), \chi(\mathcal{U})$ ), where $A(\mathcal{U})$ is the area, $L(\mathcal{U})$ is the perimeter, and $\chi(\mathcal{U})$ is the EulerPoincaré characteristic of $\mathcal{U}$. We consider here the so-called additive extension of the EulerPoincaré characteristic, which is also of primary interest in [22], i.e.

$$
\chi(\mathcal{U})=N_{\mathrm{cc}}(\mathcal{U})-N_{\mathrm{h}}(\mathcal{U}),
$$

where $N_{\mathrm{cc}}(\mathcal{U})$ is the number of connected components of $\mathcal{U}$ and $N_{\mathrm{h}}(\mathcal{U})$ is the number of holes of $\mathcal{U}$. The special case where $Q$ is degenerate and $\boldsymbol{T}(\mathcal{U})=A(\mathcal{U})$ is known as the area-interaction point process, Widom-Rowlinson model, or penetrable spheres model; see, for example, [3], [15], [22], and [45].

\subsection{General position of discs}

It becomes essential in this paper that, with probability 1, the discs defined by $\Psi$ are in general position in the following sense. Identify $\mathbb{R}^{2}$ with the hyperplane of $\mathbb{R}^{3}$ spanned by the first two coordinate axes. For each disc $b(z, r)$, define the ghost sphere $s(z, r)=\left\{y \in \mathbb{R}^{3}:\|y-z\|=r\right\}$, i.e. the hypersphere in $\mathbb{R}^{3}$ with center $z$ and radius $r$. A configuration of discs is said to be in general position if the intersection of any $k+1$ corresponding ghost spheres is either empty or a sphere of dimension $2-k$, where $k=1,2, \ldots$ Note that the intersection is assumed to be empty if $k>2$ and a sphere of dimension 0 is assumed to consist of two points. Figure 1(a) shows a configuration of discs in general position; we shall use this as a running example to illustrate forthcoming definitions.

Lemma 1. For almost all realizations of $\Psi=\left\{x_{1}, x_{2}, \ldots\right\}$, the discs $b_{1}=b\left(x_{1}\right)$ and $b_{2}=$ $b\left(x_{2}\right), \ldots$ are in general position.

Proof. By Campbell's theorem (see, for example, [41]), the mean number of sets of $k+1$ ghost spheres whose intersection is neither empty nor of dimension $2-k$ is given by

$$
\begin{aligned}
\int_{\mathbb{R}^{2}} \int_{0}^{\infty} \cdots \int_{\mathbb{R}^{2}} \int_{0}^{\infty} & \mathbf{1}\left[\bigcap_{0}^{k} s_{i} \neq \varnothing, \operatorname{dim}\left(\bigcap_{0}^{k} s_{i}\right) \neq 2-k\right] \\
& \times \frac{\prod_{0}^{k} \rho\left(z_{i}\right)}{(k+1) !} \mathrm{d} z_{0} Q\left(\mathrm{~d} r_{0}\right) \cdots \mathrm{d} z_{k} Q\left(\mathrm{~d} r_{k}\right),
\end{aligned}
$$

where $\mathbf{1}[\cdot]$ is the indicator function and $s_{i}=s\left(z_{i}, r_{i}\right)$. This integral is 0 since, for any fixed values of $r_{0}>0, \ldots, r_{k}>0$, the indicator function is 0 for Lebesgue almost all $\left(z_{0}, \ldots, z_{k}\right) \in$ $\mathbb{R}^{2(k+1)}$.

All point process models for discs considered in this paper have discs in general position: by Lemma 1 , the discs in $X$ with density (1) are in general position almost surely. 


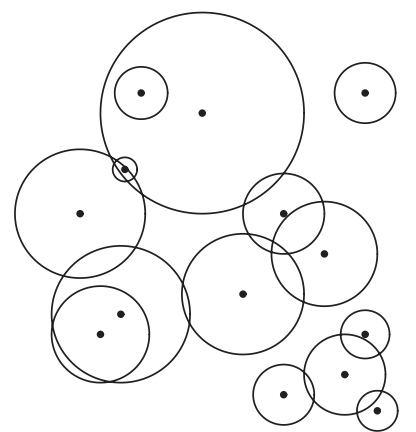

(a)

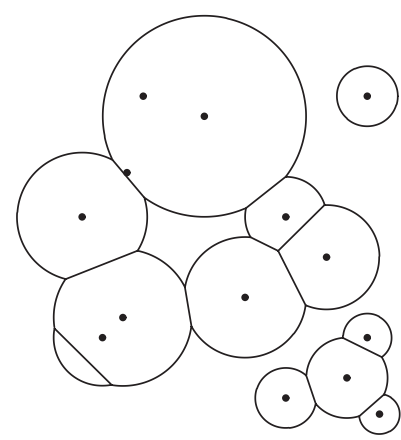

(c)

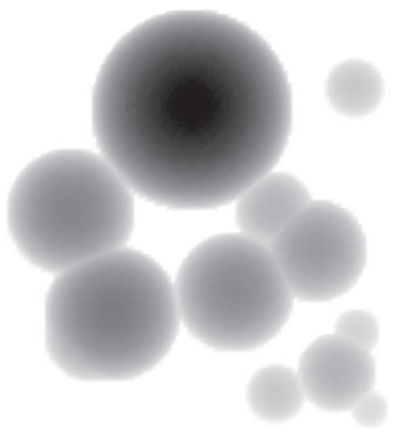

(b)

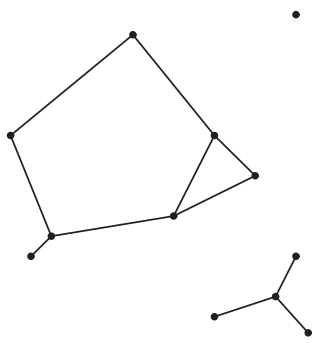

(d)

Figure 1: (a) A configuration of discs in general position. (b) The upper hemispheres as seen from above. (c) The power tessellation of the union of discs. (d) The dual graph.

\section{Power tessellation of a union of discs}

In this section we define and study the power tessellation of a union of discs

$$
u=\bigcup_{i \in I} b_{i}
$$

We assume that the discs $b_{i}, i \in I$, satisfy the general position assumption (GPA).

\subsection{Basic definitions}

In this subsection there is no need for assuming that the index set $I$ is finite, though this will be the case in subsequent sections.

For each disc $b_{i}, i \in I$, with ghost sphere $s_{i}$, let $s_{i}^{+}=\left\{\left(y_{1}, y_{2}, y_{3}\right) \in s_{i}: y_{3} \geq 0\right\}$ denote the corresponding upper hypersphere and, for $u \in b_{i}$, let $y_{i}(u)$ denote the unique point on $s_{i}^{+}$ whose orthogonal projection on $\mathbb{R}^{2}$ is $u$. The subset of $s_{i}^{+}$consisting of those points 'we can see from above' is given by

$$
C_{i}=\left\{y_{i}(u): u \in b_{i},\left\|u-y_{i}(u)\right\| \geq\left\|u-y_{j}(u)\right\| \text { whenever } u \in b_{j}, j \in I\right\},
$$

and the GPA implies that the nonempty $C_{i}$ have disjoint two-dimensional relative interiors. Thus, as illustrated in Figure 1(b), the nonempty $C_{i}$ form a tessellation (i.e. subdivision) of $\bigcup_{I} s_{i}^{+}$corresponding to the two-dimensional pieces of upper ghost spheres 'as seen from 
above'. Projecting this tessellation onto $\mathbb{R}^{2}$, we obtain a tessellation of $\mathcal{U}$; see Figure 1 (c). Below we specify this tessellation in detail.

Let $J=\left\{i \in I: C_{i} \neq \varnothing\right\}$. For $i \in I$, define the power distance of a point $u \in \mathbb{R}^{2}$ from $b_{i}=b\left(z_{i}, r_{i}\right)$ by $\pi_{i}(u)=\left\|u-z_{i}\right\|^{2}-r_{i}^{2}$, and define the power cell associated with $b_{i}$ by

$$
V_{i}=\left\{u \in \mathbb{R}^{2}: \pi_{i}(u) \leq \pi_{j}(u) \text { for all } j \in I\right\} .
$$

For distinct $i, j \in I$, define the closed halfplane $H_{i, j}=\left\{u \in \mathbb{R}^{2}: \pi_{i}(u) \leq \pi_{j}(u)\right\}$. Each $V_{i}$ is a convex polygon, since it is a finite intersection of closed halfplanes $H_{i, j}$. The power cells have disjoint interiors and, by the GPA, each $V_{i}$ is either empty or of dimension two. Consequently, the nonempty power cells $V_{i}, i \in J$, constitute a tessellation of $\mathbb{R}^{2}$ called the power diagram (or Laguerre diagram); see [1] and the references therein. In the special case where all the radii $r_{i}$ are equal we have $I=J$ and the power diagram is a Voronoi tessellation (see, for example, [29] and [36]), where each cell $V_{i}$ contains $z_{i}$ in its interior. If the radii are not equal, a power cell $V_{i}$ may not contain $z_{i}$, since $H_{i, j}$ may not contain $z_{i}$.

Let $B_{i}$ denote the orthogonal projection of $C_{i}$ on $\mathbb{R}^{2}$. By Pythagoras, for all $u \in b_{i}$, $\pi_{i}(u)+\left\|u-y_{i}(u)\right\|^{2}=0$. Consequently, for any $i, j \in I$ and $u \in b_{i} \cap b_{j}$,

$$
\left\|u-y_{i}(u)\right\| \geq\left\|u-y_{j}(u)\right\| \quad \text { if and only if } \quad \pi_{i}(u) \leq \pi_{j}(u)
$$

Thus, $B_{i}=V_{i} \cap b_{i}$. By the GPA and the one-to-one correspondence between $B_{i}$ and $C_{i}$, the collection of sets $B_{i}, i \in J$, constitutes a subdivision of $U$ into two-dimensional convex sets with disjoint interiors. We call this the power tessellation of the union of discs and denote it by $\mathcal{B}$. Furthermore, if $i \in J$, we call $B_{i}$ the power cell restricted to its associated disc $b_{i}$ (clearly, $B_{i}=\varnothing$ if $i \in I \backslash J$ ). Since $V_{i}$ may not contain $z_{i}, B_{i}$ may not contain $z_{i}$; an example of this is shown in Figure 1(c). We say that a cell $B_{i}$ is isolated if $B_{i}=b_{i}$. This means that any disc $b_{j}, j \in I$, intersecting $b_{i}$ is contained in $b_{i}$; the disc $b_{i}$ is therefore also said to be a circular clump (see [27] and the references therein).

It is illuminating to consider Figure 1 when making the following definitions. If the intersection $e_{i, j}=B_{i} \cap B_{j}$ between two cells of $\mathscr{B}$ is nonempty then $e_{i, j}=\left[u_{i, j}, v_{i, j}\right]$ is a closed line segment, where $u_{i, j}$ and $v_{i, j}$ denote the endpoints, and we call $e_{i, j}$ an interior edge of $\mathcal{B}$. The vertices of $\mathscr{B}$ are given by all endpoints of interior edges. A vertex of $\mathscr{B}$ lying on the boundary $\partial \mathcal{U}$ is called a boundary vertex, and it is called an interior vertex otherwise. Each circular arc on $\mathscr{B}$ defined by two successive boundary vertices is called a boundary edge of $\mathcal{B}$. The circle given by the boundary of an isolated cell of $\mathscr{B}$ is also called a boundary edge or sometimes an isolated boundary edge. The connected components of $\partial \mathcal{U}$ are closed curves, and each such curve is a union of certain boundary edges which either bound a hole, in which case the curve is called an inner boundary curve, or bound a connected component of $\mathcal{U}$, in which case the curve is called an outer boundary curve. A generic boundary edge of $\mathscr{B}$ is written as $\left\lfloor u_{i}, v_{i}\right\rceil$ if $B_{i} \neq b_{i}$ (a nonisolated cell), where the index means that $u_{i}$ and $v_{i}$ are boundary vertices of $B_{i}$, or as $\partial b_{i}$ if $B_{i}=b_{i}$. We order $u_{i}$ and $v_{i}$ such that $\left\lfloor u_{i}, v_{i}\right\rceil$ is the circular arc from $u_{i}$ to $v_{i}$ when $\partial b_{i}$ is considered anticlockwise.

By the GPA, any intersection among four cells of $\mathscr{B}$ is empty, each interior vertex corresponds to a nonempty intersection among three cells of $\mathscr{B}$, and exactly three edges emerge at each vertex. Note that each isolated cell has no vertices and one edge. Each interior edge $e_{i, j}$ is contained in the bisector (or power line or radical axis) of $b_{i}$ and $b_{j}$ defined by $\partial H_{i, j}=\{u \in$ $\left.\mathbb{R}^{d}: \pi_{i}(u)=\pi_{j}(u)\right\}$. This is the line perpendicular to the line joining the centers of the two 
discs and passing through the point

$$
z_{i, j}=\frac{1}{2}\left(z_{i}+z_{j}+\frac{r_{j}^{2}-r_{i}^{2}}{\left\|z_{i}-z_{j}\right\|^{2}}\left(z_{i}-z_{j}\right)\right) .
$$

We call $E_{i, j} \equiv \partial H_{i, j} \cap b_{i}=\partial H_{i, j} \cap b_{j}$ the chord of $b_{i} \cap b_{j}$. Obviously, $e_{i, j} \subseteq E_{i, j}$.

The dual graph $\mathscr{D}$ to $\mathscr{B}$ has nodes equal to the centers $z_{i}, i \in J$, of discs generating nonempty cells, and each edge of $\mathscr{D}$ is given by two vertices $z_{i}$ and $z_{j}$ such that $e_{i, j} \neq \varnothing$. See Figure 1(d). Note that there is a one-to-one correspondence between the edges of $\mathscr{D}$ and the interior edges of $\mathscr{B}$.

\subsection{Construction}

We construct the power tessellation of a finite union of discs by successively adding the discs one by one, keeping track of old and new edges and whether each disc generates a nonempty cell or not. The updates are local in some sense and used in the 'birth part' of the MCMC algorithm in Section 4.7. For details, see Section A.1.

\section{Results for exponential family models}

In this section we study exponential family models for the point process $X$ as specified by the density $f(x)$ in (1), assuming that the canonical sufficient statistic $\boldsymbol{T}\left(\mathcal{U}_{x}\right)$ is a linear combination of one or more of the geometric characteristics introduced in the following paragraph. We let $\operatorname{supp}(Q)$ denote the support of $Q$, let

$$
\Omega=\{(z, r) \in S \times(0, \infty): \rho(z)>0, r \in \operatorname{supp}(Q)\}
$$

denote the support of the intensity measure of the reference Poisson process $\Psi$, and let $\mathcal{N}$ denote the set of all finite subsets $x$ (also called finite configurations) of $\Omega$ so that the discs given by $x$ are in general position. By Lemma $1, X \in \mathcal{N}$ with probability 1 . For ease of exposition, we assume that all realizations of $X$ are in $\mathcal{N}$, and set $f(x)=0$ if $x \notin \mathcal{N}$.

We let $\boldsymbol{T}(x)$ be given by one or more of the following characteristics of $\mathcal{U}=U_{x}$ if $x \in \mathcal{N}$ : the area $A=A(\mathcal{U})$, the perimeter $L=L(\mathcal{U})$, the Euler-Poincaré characteristic $\chi=\chi(U)$, the number of isolated cells $N_{\mathrm{ic}}=N_{\mathrm{ic}}(\mathcal{U})$, the number of connected components $N_{\mathrm{cc}}=N_{\mathrm{cc}}(U)$, the number of holes $N_{\mathrm{h}}=N_{\mathrm{h}}(\mathcal{U})$, the number of boundary edges (including isolated boundary edges) $N_{\text {be }}=N_{\text {be }}(\mathcal{U})$, and the number of boundary vertices $N_{\mathrm{bv}}=N_{\mathrm{bv}}(\mathcal{U})$. In the general case

$$
\boldsymbol{T}=\left(A, L, \chi, N_{\mathrm{h}}, N_{\mathrm{ic}}, N_{\mathrm{bv}}\right)
$$

with corresponding canonical parameter $\boldsymbol{\theta}=\left(\theta_{1}, \ldots, \theta_{6}\right)$, and we then call $X$ the $\boldsymbol{T}$-interaction process. If, for example, $\theta_{2}=\cdots=\theta_{6}=0$, we set $\boldsymbol{T}=A$ and refer then to the $A$-interaction process. Similarly, for the $L$-interaction process, we have $\theta_{1}=0$ and $\theta_{3}=\cdots=\theta_{6}=0$, for the $(A, L)$-interaction process, we have $\theta_{3}=\cdots=\theta_{6}=0$, and so on. A quermass-interaction process [22] is the special case $\boldsymbol{T}=(A, L, \chi)$ and $\theta_{4}=\theta_{5}=\theta_{6}=0$. Note that (4) specifies $N_{\mathrm{cc}}=\chi+N_{\mathrm{h}}$ and $N_{\mathrm{be}}=N_{\mathrm{ic}}+N_{\mathrm{bv}}$; cf. Lemma 2, below. Thus, a continuum random-cluster model [15], [23], [28] is the special case $\boldsymbol{T}=N_{\mathrm{cc}}, \theta_{1}=\theta_{2}=\theta_{5}=\theta_{6}=0$, and $\theta_{3}=\theta_{4}$. Although natural in terms of the power diagram, we may question how useful it is to include $N_{\text {ic }}$ and $N_{\text {bv }}$ in (4), since in practice grains may only approximately be discs and only a digital image is observed, where the resolution makes it difficult to identify circular structures. Moreover, 
the interplay of $\left(\theta_{5}, \theta_{6}\right)$ with other parameters seems complicated; we return to this aspect at the end of Section 4.2.

\subsection{Exponential family structure}

Let

$$
\Theta=\left\{\left(\theta_{1}, \ldots, \theta_{6}\right) \in \mathbb{R}^{6}: \int \exp \left(\pi \theta_{1} r^{2}+2 \pi \theta_{2} r\right) Q(\mathrm{~d} r)<\infty\right\} .
$$

Note that $(-\infty, 0]^{2} \times \mathbb{R}^{4} \subseteq \Theta$ and that $\Theta=\mathbb{R}^{6}$ if $\operatorname{supp}(Q)$ is bounded. The following proposition states that, under a weak condition on $(S, \rho, Q)$, the exponential family density has $\Theta$ as its full parameter space and $\boldsymbol{T}$ in (4) as its minimal canonical sufficient statistic (for details on exponential family properties, see [5]).

Proposition 1. Suppose that $S$ contains a set $D=b\left(u, R_{1}\right) \backslash b\left(u, R_{2}\right)$, where $\infty>R_{1}>$ $R_{2}>0, \rho(z)>0$ for all $z \in D$, and $Q\left(\left(0, R_{2}\right]\right)>0$. Then the point process densities

$$
\begin{gathered}
f_{\boldsymbol{\theta}}(x)=\frac{1}{c_{\boldsymbol{\theta}}} \exp \left(\theta_{1} A\left(\mathcal{u}_{x}\right)+\theta_{2} L\left(u_{x}\right)+\theta_{3} \chi\left(u_{x}\right)+\theta_{4} N_{\mathrm{h}}\left(u_{x}\right)\right. \\
\left.+\theta_{5} N_{\mathrm{ic}}\left(u_{x}\right)+\theta_{6} N_{\mathrm{bv}}\left(u_{x}\right)\right)
\end{gathered}
$$

with $x \in \mathcal{N}$ and $\boldsymbol{\theta}=\left(\theta_{1}, \ldots, \theta_{6}\right) \in \Theta$ constitute a regular exponential family model.

Proof. Recall that an exponential family model is regular if it is full and of minimal form [5]. Later in Proposition 6, we verify that $f_{\boldsymbol{\theta}}$ is well defined if and only if $\boldsymbol{\theta} \in \Theta$, so the model is full. Let $\Psi_{S}$ denote the restriction of $\Psi$ to $S \times(0, \infty)$. Since $\Theta \supseteq(-\infty, 0]^{2} \times \mathbb{R}^{4}$ is of full dimension 6 and since there is a one-to-one linear correspondence between $\boldsymbol{T}$ in (4) and ( $A, L, N_{\mathrm{cc}}, N_{\mathrm{ic}}, N_{\mathrm{bv}}, N_{\mathrm{h}}$ ), the model is on minimal form if the statistics $A, L, N_{\mathrm{ic}}, N_{\mathrm{cc}}, N_{\mathrm{bv}}$, and $N_{\mathrm{h}}$ are affinely independent with probability 1 with respect to $\Psi_{S}$ (see [5]). In other words, the model is on minimal form if, for any $\left(\alpha_{0}, \ldots, \alpha_{6}\right) \in \mathbb{R}^{7}$, with probability 1 ,

$$
\begin{gathered}
\alpha_{1} A\left(U_{\Psi_{S}}\right)+\alpha_{2} L\left(U_{\Psi_{S}}\right)+\alpha_{3} N_{\mathrm{ic}}\left(\mathcal{U}_{\Psi_{S}}\right)+\alpha_{4} N_{\mathrm{cc}}\left(U_{\Psi_{S}}\right)+\alpha_{5} N_{\mathrm{bv}}\left(U_{\Psi_{S}}\right) \\
+\alpha_{6} N_{\mathrm{h}}\left(U_{\Psi_{S}}\right)=\alpha_{0} \quad \Longrightarrow \quad \alpha_{0}=\cdots=\alpha_{6}=0 .
\end{gathered}
$$

We verify this, using the condition on $(S, \rho, Q)$ imposed in the proposition, and considering realizations of $\Psi_{S}$ as described below, where these realizations consist of configurations of discs with centers in $D$ and radius less than or equal to $R_{2}$. For such configurations, given by either one disc, two nonoverlapping discs, or two overlapping discs, and if $\alpha_{5}=\alpha_{6}=0$, we immediately obtain (7). Extending this to situations where only $\alpha_{6}=0$ and where we have three discs with pairwise overlap but no common intersection, we also immediately obtain (7) and the set consisting of such configurations where $N_{\mathrm{h}}\left(U_{\Psi_{S}}\right)=0$ has a positive probability. The condition on $(S, \rho, Q)$ also allows us, with a positive probability, to construct a set of realizations where $N_{\mathrm{h}}\left(\mathcal{U}_{\Psi_{S}}\right)=1$, namely by considering sequences of discs which only overlap pairwise and which form a single connected component. Thereby, for any $\left(\alpha_{0}, \ldots, \alpha_{6}\right) \in \mathbb{R}^{7}$, with probability $1,(7)$ is seen to hold.

\subsection{Interpretation of parameters}

In this subsection we discuss the meaning of the parameters $\theta_{1}, \ldots, \theta_{6}$ in the $\boldsymbol{T}$-interaction process, (6). 
We first recall the definition of the Papangelou conditional intensity $\lambda(x, v)$ for a general finite point process $X \subset S \times(0, \infty)$ with a hereditary density $f$ with respect to the distribution of $\Psi$ (see [34] and the references therein). For all finite configurations $x \subset S \times(0, \infty)$ and all discs $v=(z, r) \in S \times(0, \infty) \backslash x$, the hereditary condition means that $f(x)>0$ whenever $f(x \cup\{v\})>0$, and, by definition,

$$
\lambda(x, v)= \begin{cases}\frac{f(x \cup\{v\})}{f(x)} & \text { if } f(x)>0, \\ 0 & \text { otherwise. }\end{cases}
$$

This is in a one-to-one correspondence with the density $f$, and has the interpretation that $\lambda(x, v) \rho(z) \mathrm{d} z Q(\mathrm{~d} r)$ is the conditional probability of $X$ having a disc with center in an infinitesimal region containing $z$ and of size $\mathrm{d} z$ and radius in an infinitesimal region containing $r$ and of size $\mathrm{d} r$, given that the rest of $X$ is $x$.

For functionals $W=A, L, \ldots$, define $W(x, v)=W\left(U_{x \cup\{v\}}\right)-W\left(U_{x}\right)$. The $\boldsymbol{T}$-interaction process, (6), has a hereditary density with Papangelou conditional intensity

$$
\begin{gathered}
\lambda_{\theta}(x, v)=\exp \left(\theta_{1} A(x, v)+\theta_{2} L(x, v)+\theta_{3} \chi(x, v)+\theta_{4} N_{\mathrm{h}}(x, v)\right. \\
\left.+\theta_{5} N_{\mathrm{ic}}(x, v)+\theta_{6} N_{\mathrm{bv}}(x, v)\right)
\end{gathered}
$$

if $x \cup\{v\} \in \mathcal{N}$, and $\lambda_{\theta}(x, v)=0$ otherwise. Note that $\mathcal{N}$ is hereditary, meaning that $x \in \mathcal{N}$ implies that $y \in \mathcal{N}$ if $y \subset x$. The process $X$ is said to be attractive if

$$
\lambda_{\theta}(x, v) \geq \lambda_{\theta}(y, v) \quad \text { whenever } y \subset x \text { and } x \in \mathcal{N}
$$

and repulsive if

$$
\lambda_{\theta}(x, v) \leq \lambda_{\theta}(y, v) \quad \text { whenever } y \subset x \text { and } x \in \mathcal{N} .
$$

Note that, since quermass integrals are additive,

$$
\begin{gathered}
A(x, v)=A\left(b_{v}\right)-A\left(b_{v} \cap U_{x}\right), \quad L(x, v)=L\left(b_{v}\right)-L\left(b_{v} \cap U_{x}\right), \\
\chi(x, v)=1-N_{\mathrm{h}}\left(b_{v} \cap U_{x}\right) .
\end{gathered}
$$

Proposition 2. The following assertions hold:

(a) the A-interaction process is attractive if $\theta_{1}<0$ and repulsive if $\theta_{1}>0$;

(b) under weak conditions, for example, if S contains an open disc, the L-interaction process is neither attractive nor repulsive if $\theta_{2} \neq 0$;

(c) under other weak conditions, basically meaning that $S$ is not too small compared to $\inf \operatorname{supp}(Q)$ (as exemplified in the proof), the $W$-interaction processes with $W=\chi, N_{\mathrm{h}}$, $N_{\mathrm{ic}}, N_{\mathrm{bv}}$ are neither attractive nor repulsive if $\theta_{i} \neq 0, i=3,4,5,6$;

(d) under similar weak conditions as in (c), the continuum random-cluster model (i.e. the $N_{\mathrm{cc}}$-interaction process, where $\theta_{3}=\theta_{4}$ and $\left.\theta_{1}=\theta_{2}=\theta_{5}=\theta_{6}=0\right)$ is neither attractive nor repulsive if $\theta_{3} \neq 0$.

Proof. From (11), part (a) follows immediately, which is a well-known result [3]. We have $L\left(b_{v} \cap U_{x_{1}}\right)>0=L\left(b_{v} \cap U_{\varnothing}\right)$ if $b_{v} \cap b_{x_{1}} \neq \varnothing$. This provides a simple example where $\lambda_{\theta_{2}}(x, v)$ is decreasing or increasing in $x$ if $\theta_{2}>0$ or $\theta_{2}<0$, respectively. On the other hand, if $S$ contains an open disc, we may obtain the opposite case. Figure 2(a) shows such an example, 


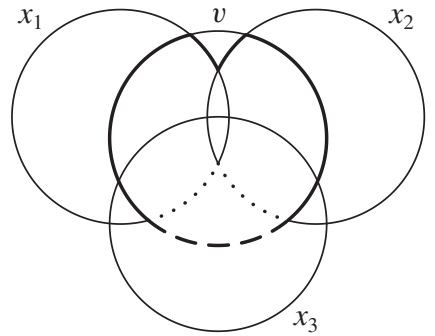

(a)

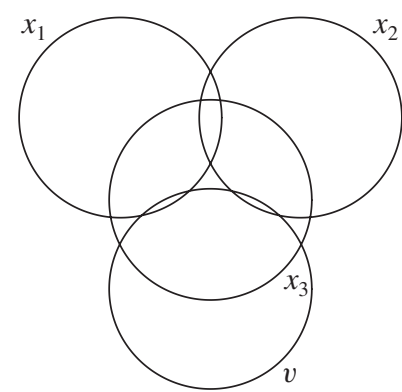

(b)

FIGURE 2: Examples of four discs of equal radii. (a) When we add $x_{3}$ to $\left\{x_{1}, x_{2}\right\}$, the dotted arcs disappear and the dashed arc appears, so $L\left(b_{v} \cap \mathcal{U}_{\left\{x_{1}, x_{2}, x_{3}\right\}}\right)<L\left(b_{v} \cap \mathcal{U}_{\left\{x_{1}, x_{2}\right\}}\right)$. (b) $N_{\mathrm{bv}}\left(\left\{x_{1}, x_{2}\right\}, v\right)=4$ and $N_{\text {bv }}\left(\left\{x_{1}, x_{2}, x_{3}\right\}, v\right)=2$.

with four discs of equal radii, where the four centers of the discs can be made arbitrarily close and where $L\left(b_{v} \cap U_{x_{1}, x_{2}, x_{3}}\right)<L\left(b_{v} \cap \mathcal{U}_{x_{1}, x_{2}}\right)$. Thereby part (b) is verified.

To verify parts (c) and (d), we again consider discs $b_{v}, b_{x_{1}}, b_{x_{2}}, \ldots$ of equal radii, since it may be possible that $Q$ is degenerate.

Suppose that $b_{v} \cap b_{x_{1}}=\varnothing, b_{v} \cap b_{x_{2}} \neq \varnothing$, and $b_{x_{1}} \cap b_{x_{2}} \neq \varnothing$, and let $x=\left\{x_{1}, x_{2}\right\}$. Then $\chi(y, v)=2$ and $\chi(x, v)=1$ if $y=\left\{x_{1}\right\}$, while $\chi(y, v)=1$ and $\chi(x, v)=2$ if $y=\left\{x_{2}\right\}$. Since $\chi=N_{\text {cc }}$ in these examples, we obtain part (c) in the case of the $\chi$-interaction process and part (d) in the case of the $N_{\mathrm{cc}}$-interaction process.

Suppose that $b_{v}, b_{x_{1}}$, and $b_{x_{2}}$ have no common intersection, but that each pair of discs are overlapping, i.e. they form a hole. If $y=\left\{x_{1}, x_{2}\right\}$ and the hole disappears when we consider $x=\left\{x_{1}, x_{2}, x_{3}\right\}$ then $N_{\mathrm{h}}(y, v)=1$ and $N_{\mathrm{h}}(x, v)=0$. Note that $N_{\mathrm{bv}}(y, v)=4$ and it may be possible that $N_{\mathrm{bv}}(x, v)=2$, as exemplified in Figure 2(b). On the other hand, if $y=\left\{x_{1}\right\}$ and $x=\left\{x_{1}, x_{2}\right\}$ then $N_{\mathrm{h}}(y, v)=0, N_{\mathrm{h}}(x, v)=1, N_{\mathrm{bv}}(y, v)=2$, and $N_{\mathrm{bv}}(x, v)=4$. Hence, we have established part (c) in the case of the $N_{\mathrm{h}}$-interaction and $N_{\mathrm{bv}}$-interaction processes.

Finally, the case of the $N_{\text {ic }}$-interaction process in part (c) follows simply by considering two overlapping discs and two disjoint discs.

Thus, in terms of the 'local characteristic' $\lambda_{\theta}(x, v)$, we can easily interpret the importance of the parameter $\theta_{1}$ in the $A$-interaction process and also that of $\theta_{2}$ in the $L$-interaction process provided that $Q$ is degenerate, while the role of the parameters in the other processes is less clear. Their meaning is better understood in 'global terms' and by simulation studies. In comparison with the reference Poisson process, the $A$-interaction processes with $\theta_{1}>0$ and $\theta_{1}<0$ tend to produce realizations with a larger and smaller area $A\left(U_{x}\right)$, respectively; see Figure 3(b) and (c), and similarly for the $W$-interaction process with $W=L, \chi, N_{\mathrm{h}}, N_{\mathrm{ic}}, N_{\mathrm{bv}}, N_{\mathrm{cc}}$. For models with two or more parameters, the interpretation is more complicated and depends not only on the signs of the parameters but also on how large the parameters are, and it is, for example, possible to obtain rather similar realizations for different combinations of the parameters. See Figures 5-8 in our research report [31]. As an illustration, Figure 3(d)-(f) show realizations of the $\left(A, L, N_{\mathrm{cc}}\right)$-interaction process with $\left(\theta_{1}, \theta_{2}\right)=(0.6,-1)$ and different positive values of $\theta_{3}=\theta_{4}$. Here the effect of increasing $\theta_{3}=\theta_{4}$ should be clear and, at least with respect to the characteristic $A$, the process corresponding to Figure 3(e) seems to have some similarity to the $A$-interaction process corresponding to Figure 3(c), although the sign of $\theta_{1}$ is different in the two processes and the connected components in the two realizations look very different. 


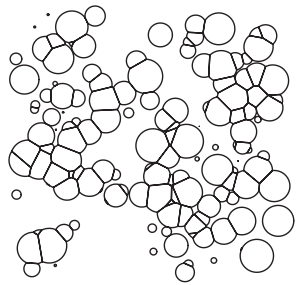

(a)

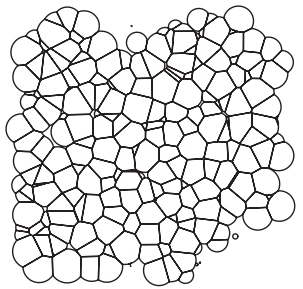

(d)

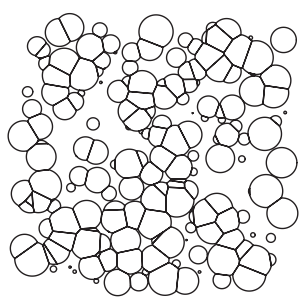

(b)

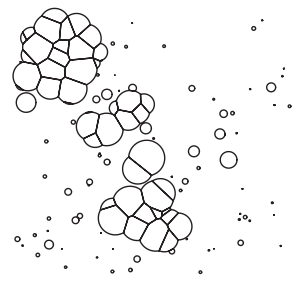

(e)

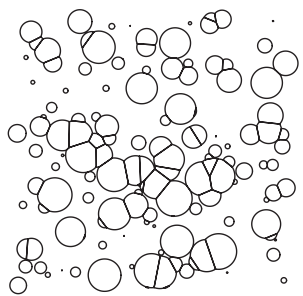

(c)

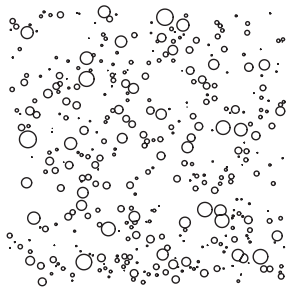

(f)

Figure 3: (a) The power tessellation of a realization of the reference Poisson process with $Q$ the uniform distribution on the interval $[0,2], \rho(u)=0.2$ on a rectangular region $S=[0,30] \times[0,30]$, and $\rho(u)=0$ outside $S$; simulated realizations of the $A$-interaction process with (b) $\theta_{1}=0.1$ or (c) $\theta_{1}=-0.1$; and simulated realizations of the $\left(A, L, N_{\mathrm{cc}}\right)$-interaction process with $\left(\theta_{1}, \theta_{2}\right)=(0.6,-1)$ and (d) $\theta_{3}=\theta_{4}=$ 1 , (e) $\theta_{3}=\theta_{4}=2$, or (f) $\theta_{3}=\theta_{4}=5$.

\subsection{Geometric characteristics and inclusion-exclusion formulae}

Lemmas 2 and 3, below, concern various useful relations between certain geometric characteristics of the union $\mathcal{U}=U_{x}$ and of its power tessellation $\mathscr{B}=\mathscr{B}_{x}$, assuming that $x \in \mathcal{N}$. Among other things, the results become useful in connection to the computation of geometric characteristics in Section 4.4 and for the sequential constructions considered in Sections 3.2 and 4.7 and Appendix A.

Define the following characteristics of $\mathscr{B}=\mathscr{B}_{x}$ : the number of nonempty cells $N_{\mathrm{c}}=N_{\mathrm{c}}(\mathscr{B})$, the number of interior edges $N_{\text {ie }}=N_{\text {ie }}(\mathscr{B})$, the number of edges $N_{\mathrm{e}}=N_{\text {be }}+N_{\text {ie }}$, the number of interior vertices $N_{\mathrm{iv}}=N_{\mathrm{iv}}(\mathscr{B})$, and the number of vertices $N_{\mathrm{v}}=N_{\mathrm{bv}}+N_{\mathrm{iv}}$. These statistics do not appear in the specification, (4), since they cannot be determined from $\mathcal{U}$; they can be determined only from $\mathscr{B}$. Furthermore, let $N=n(x)$ denote the number of discs.

Lemma 2. We have

$$
N_{\text {ic }} \leq N_{\mathrm{cc}} \leq N_{\mathrm{c}} \leq N, \quad N_{\mathrm{bv}}=2 N_{\mathrm{ie}}-3 N_{\mathrm{iv}}
$$

and

$$
\chi=N_{\mathrm{cc}}-N_{\mathrm{h}}=N_{\mathrm{c}}-N_{\mathrm{ie}}+N_{\mathrm{iv}} .
$$

If $N_{\mathrm{c}} \geq 2$ and $N_{\mathrm{cc}}=1$ then

$$
N_{\mathrm{be}}=N_{\mathrm{bv}} \leq 2 N_{\mathrm{ie}}, \quad 3 N_{\mathrm{v}}=2 N_{\mathrm{e}}
$$

If $N_{\mathrm{c}} \geq 3$ and $N_{\mathrm{cc}}=1$ then

$$
N_{\text {ie }} \leq 3 N_{\mathrm{c}}-6
$$




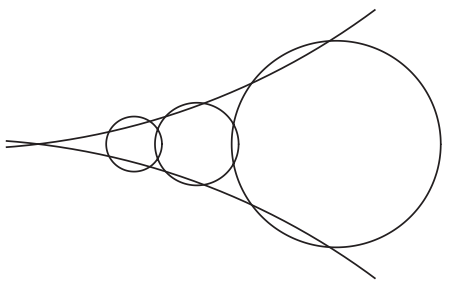

FIGURE 4: A configuration of five discs with exactly $2 N_{\mathrm{c}}-5$ holes.

Moreover,

$$
N_{\mathrm{bv}} \leq 6 N
$$

and

$$
N_{\mathrm{h}}=0 \quad \text { if } N_{\mathrm{c}} \leq 2, \quad N_{\mathrm{h}} \leq 2 N_{\mathrm{c}}-5 \quad \text { if } N_{\mathrm{c}} \geq 3 .
$$

Proof. The inequalities in (12) clearly hold, and the identity in (12) follows from a simple counting argument, using the facts that each interior edge has two endpoints and that exactly three interior edges emerge at each interior vertex.

The first identity in (13) is just the definition (3), and the second identity follows from Euler's formula.

Assuming that $N_{\mathrm{c}} \geq 2$ and $N_{\mathrm{cc}}=1$, (14) follows from simple counting arguments, using firstly the fact that exactly two boundary edges emerge at each boundary vertex, secondly the simple fact that $N_{\mathrm{bv}} \leq N_{\mathrm{v}}$, and thirdly the fact that exactly three edges emerge at each vertex.

To verify (15), consider the dual graph $\mathscr{D}$. Since we assume that $N_{\mathrm{c}} \geq 3$ and $N_{\mathrm{cc}}=1, \mathscr{D}$ has $N_{\text {ie }}$ edges and $N_{\mathrm{c}}$ vertices, and so, by planar graph theory [46], since $\mathscr{D}$ is a connected graph without multiple edges, the number of dual edges is bounded by $3 N_{\mathrm{c}}-6$.

To verify (16), note that $N_{\mathrm{bv}} \leq 2 N_{\mathrm{ie}}$; cf. (12). Using (15) and considering a sum over all components, we find that $N_{\text {ie }}$ is bounded above by the number of components with two cells plus three times the number of components with three or more cells. Consequently, $N_{\mathrm{bv}} \leq 6 \mathrm{~N}$.

Finally, to verify (17), note that $N_{\mathrm{h}}$ is given by the sum of the number of holes of all connected components of $U$, and a connected component consisting of one or two power cells has no holes, so it suffices to consider the case where $N_{\mathrm{cc}}=1$ and $N_{\mathrm{c}} \geq 3$. Then, by (13), $N_{\mathrm{h}}$ is bounded above by $1-\left(N_{\mathrm{c}}-N_{\mathrm{ie}}\right)$, which in turn, by (15), is bounded above by $2 N_{\mathrm{c}}-5$.

Equation (17) is a main result in [22]. Our proof of (17) is much simpler and shorter, demonstrating the usefulness of the power tessellation and its dual graph. The upper bound in (17) can be obtained for any three or more discs. If $x$ consists of three discs $b_{1}, b_{2}$, and $b_{3}$ such that $b_{i} \cap b_{j} \neq \varnothing$ for $1 \leq i<j \leq 3$ and $b_{1} \cap b_{2} \cap b_{3}=\varnothing$, then $N_{\mathrm{h}}=1$ and $N_{\mathrm{c}}=3$, so $N_{\mathrm{h}}=2 N_{\mathrm{c}}-5$. Furthermore, we may add a fourth, fifth, ... disc, where each added disc generates two new holes - as illustrated in Figure 4 in the case of five discs-whereby $N_{\mathrm{c}}=3,4, \ldots$ and $N_{\mathrm{h}}=2 N_{\mathrm{c}}-5$ in each case.

Kendall et al. [22] noticed the following inclusion-exclusion formula for the functionals $W=A, L, \chi:$

$$
W\left(u_{x}\right)=\sum_{1}^{n} W\left(b_{i}\right)-\sum_{1 \leq i<j \leq n} W\left(b_{i} \cap b_{j}\right)+\cdots+(-1)^{n-1} W\left(b_{1} \cap \cdots \cap b_{n}\right),
$$

where the sums involve $2^{n}-1$ terms. Using the power tessellation, inclusion-exclusion formulae with much fewer terms are given by (12) and (13) for $\chi$ and $N_{\mathrm{bv}}$ and, by Lemma 3 , 
below, for $A$ and $L$. In Lemma $3, I_{1}(x), I_{2}(x)$, and $I_{3}(x)$ denote index sets corresponding to nonempty cells, interior edges, and interior vertices of $\mathscr{B}_{x}$, respectively. For later use in Section 4.5, note that $I_{1}(x)$ and $I_{2}(x)$ correspond to the cliques in the dual graph $\mathscr{D}_{x}$ consisting of 1 and 2 nodes, respectively, while $I_{3}(x)$ corresponds to the subset of 3-cliques $\{i, j, k\} \in \mathscr{D}_{x}$ with $b_{i} \cap b_{j} \cap b_{k} \neq \varnothing$ (i.e. $b_{i} \cup b_{j} \cup b_{k}$ has no hole). Note that if $\{i, j, k\} \in \mathscr{D}_{x}$ then $b_{i} \cap b_{j} \cap b_{k} \neq \varnothing$ if and only if $E_{i, j} \cap E_{i, k} \neq \varnothing$, where the latter property is easily checked.

Lemma 3. The following inclusion-exclusion formulae hold for the area and perimeter of the union of discs:

$$
\begin{aligned}
A\left(u_{x}\right) & =\sum_{i \in I_{1}(x)} A\left(b_{i}\right)-\sum_{\{i, j\} \in I_{2}(x)} A\left(b_{i} \cap b_{j}\right)+\sum_{\{i, j, k\} \in I_{3}(x)} A\left(b_{i} \cap b_{j} \cap b_{k}\right) \\
& =\sum_{i \in I_{1}(x)} A\left(B_{i}\right)
\end{aligned}
$$

and

$$
\begin{aligned}
L\left(u_{x}\right) & =\sum_{i \in I_{1}(x)} L\left(b_{i}\right)-\sum_{\{i, j\} \in I_{2}(x)} L\left(b_{i} \cap b_{j}\right)+\sum_{\{i, j, k\} \in I_{3}(x)} L\left(b_{i} \cap b_{j} \cap b_{k}\right) \\
& =\sum_{e \text { boundary edge of } \mathcal{B}_{x}} L(e) .
\end{aligned}
$$

Proof. Equations (19) and (21) are due to Theorem 6.2 of [10], while (20) and (22) follow immediately.

Edelsbrunner [10] established extensions to $\mathbb{R}^{d}$ of the inclusion-exclusion formulae given by the second identities in (12), (19), and (21). Note that we cannot replace the sums in (19) by sums over all discs, pairs of discs, and triplets of discs from $x$.

\subsection{Local calculations}

For calculating the area and perimeter, the inclusion-exclusion formulae (20) and (22) appear to be more suited than (19) and (21) when the computations are done in combination with the sequential constructions considered in Sections 3.2 and 4.7 and Appendix A. Note that we need only do 'local computations'.

For example, suppose that we are given the power tessellation $\mathscr{B}^{\text {old }}$ of $\mathcal{U}^{\text {old }}=\bigcup_{1}^{n-1} b_{i}$ and that we add a new disc $b_{n}$. When constructing the new power tessellation $\mathscr{B}^{\text {new }}$ of $\mathcal{U}^{\text {new }}=$ $\bigcup_{1}^{n} b_{i}$, we need only consider the new set $B_{n}$ and the old cells in $\mathscr{B}^{\text {old }}$ which are neighbors to $B_{n}$ with respect to the dual graph of $\mathscr{B}^{\text {new }}$ (see Section A.1). Similarly, when a disc is deleted and the new tessellation is constructed, we need only do local computations with respect to the discs intersecting the disc which is deleted (see Section A.2); we study this neighbor relation given by overlapping discs in Section 4.5. Moreover, local computations are only needed when calculating $N_{\text {ic }}$ and $N_{\text {bv. }}$.

In order to calculate $\left(\chi, N_{\mathrm{h}}\right)$ or, equivalently, $\left(N_{\mathrm{cc}}, N_{\mathrm{h}}\right)$, we could keep track on the inner and outer boundary curves in our sequential constructions, using a clockwise and anticlockwise orientation for the two different types of boundary curves. However, in our MCMC simulation codes, we found it easier to keep track on $N_{\mathrm{c}}, N_{\mathrm{ie}}, N_{\mathrm{iv}}$, and $N_{\mathrm{cc}}$, and thereby obtain $\chi$ by the second equality in (13) and, hence, obtain $N_{\mathrm{h}}$ by the first inequality in (13). In either case this is another kind of local computation, where the relevant neighbor relation is the connected component relation studied in Section 4.5. 
Finally, let us explain in more detail how we can find the area $A$. We can easily determine the total area of all isolated cells of $\mathscr{B}$. Suppose that $B_{i}$ is a nonempty, nonisolated cell of $\mathscr{B}$. Let $c_{i}$ denote the arithmetic average of the vertices of $B_{i}$. Then $c_{i} \in B_{i}$, since $B_{i}$ is convex. For any three points $c, u, v \in \mathbb{R}^{2}$, let $\Delta(c, u, v)$ denote the triangle with vertices $c, u$, and $v$. If $\lfloor u, v\rceil$ is a boundary edge of $B_{i}$, let $\Gamma(u, v)$ denote the cap of $b_{i}$ bounded by the arc $\lfloor u, v\rceil$ and the line segment $[u, v]$. Then the area of $B_{i}$ is the sum of areas of all triangles $\Delta\left(c_{i}, u, v\right)$, where $u$ and $v$ are defining an (interior or boundary) edge of $B_{i}$, plus the sum of areas of all caps $\Gamma(u, v)$, where $u$ and $v$ are defining a boundary edge of $B_{i}$.

\subsection{Markov properties}

The various Markov point process models considered in this subsection are either specified by a local Markov property in terms of the Papangelou conditional intensity or by a particular form of the density given by a Hammersley-Clifford-type theorem [2], [38]. In particular, we show that it is useful to view the $\boldsymbol{T}$-interaction process (6) as a connected component Markov point process, where we show how a spatial Markov property becomes useful for handling edge effects. Throughout Sections 4.5.1-4.5.5, we let $x \in \mathcal{N}$.

4.5.1. Local Markov property in terms of the overlap relation. Consider the overlap relation ' $\sim$ ' defined on $S \times(0, \infty)$ by $u \sim v$ if and only if $b(u) \cap b(v) \neq \varnothing$. The $\boldsymbol{T}$-interaction process is said to be Markov with respect to the overlap relation if $\lambda_{\theta}(x, v)$ depends only on $x$ through $\{u \in x: u \sim v\}$, i.e. the neighbors in $x$ to $v$ [2], [38], [42]. Kendall et al. [22] observed that the quermass-interaction process is Markov with respect to the overlap relation. The following proposition generalizes this result.

Proposition 3. The $\boldsymbol{T}$-interaction process with density (6) is Markov with respect to the overlap relation if and only if $\theta_{4}=\theta_{5}=0$.

Proof. In other words, with respect to the overlap relation, we have to verify that the $A$-interaction, $L$-interaction, $\chi$-interaction, and $N_{\mathrm{bv}}$-interaction processes are Markov, while the $N_{\mathrm{h}}$-interaction and $N_{\text {ic }}$-interaction processes are not Markov. It follows immediately from (8) and (11) that the $A$-interaction, $L$-interaction, and $\chi$-interaction processes are Markov, and Figures 5 and 6 respectively show that the $N_{\mathrm{h}}$-interaction and $N_{\text {ic }}$-interaction processes are not Markov. If $w$ is a boundary vertex of $U_{x}$ but not of $U_{x \cup\{v\}}$ then $w$ is contained in the disc $v$. If instead $w$ is a boundary vertex of $U_{x \cup\{v\}}$ but not of $U_{x}$ then $w$ is given by the intersection of the boundaries of $v$ and an $x$-disc. Consequently, $N_{\mathrm{bv}}(x, v)=N_{\mathrm{bv}}\left(\mathcal{U}_{x \cup\{v\}}\right)-N_{\mathrm{bv}}\left(U_{x}\right)$ depends on $x$ only through $\{u \in x: u \sim v\}$, so the $N_{\mathrm{bv}}$-interaction process is Markov. This completes the proof.
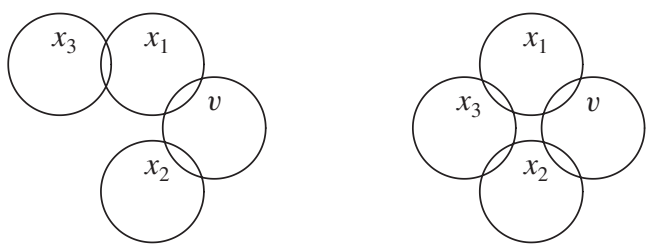

FIGURE 5: An example showing that the $N_{\mathrm{h}}$-interaction process is not Markov with respect to the overlap relation: both $N_{\mathrm{h}}(x, v)=0($ left $)$ and $N_{\mathrm{h}}(x, v)=1$ (right) depend on the disc $x_{3}$, which is not overlapping the disc $v$ 

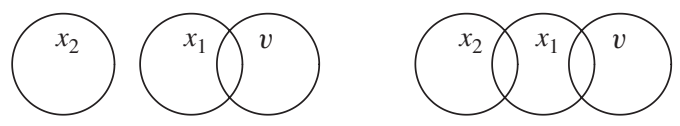

FiguRE 6: An example showing that the $N_{\text {ic }}$-interaction process is not Markov with respect to the overlap relation: both $N_{\mathrm{ic}}(x, v)=-1$ (left) and $N_{\mathrm{ic}}(x, v)=0$ (right) depend on the disc $x_{2}$, which is not overlapping the disc $v$.

As noted in [22], using the inclusion-exclusion formula (18), the Hammersley-Clifford representation [38] of the quermass-interaction process is

$$
f_{\left(\theta_{1}, \theta_{2}, \theta_{3}\right)}(x)=\prod_{y \subseteq x} \phi_{\left(\theta_{1}, \theta_{2}, \theta_{3}\right)}(y),
$$

where the interaction function is given by

$$
\phi_{\left(\theta_{1}, \theta_{2}, \theta_{3}\right)}(x)=\exp \left((-1)^{n}\left(\theta_{1} A\left(\bigcap_{1}^{n} b_{i}\right)+\theta_{2} L\left(\bigcap_{1}^{n} b_{i}\right)+\theta_{3} \chi\left(\bigcap_{1}^{n} b_{i}\right)\right)\right)
$$

for nonempty $x=\left\{\left(z_{1}, r_{1}\right), \ldots,\left(z_{n}, r_{n}\right)\right\}$ and $\phi_{\left(\theta_{1}, \theta_{2}, \theta_{3}\right)}(\varnothing)=1 / c_{\left(\theta_{1}, \theta_{2}, \theta_{3}\right)}$. However, for at least two reasons, it is the density in (6) of the quermass-interaction process rather than the Hammersley-Clifford representation (23) which seems appealing. First, the process has interactions of all orders, since $\log \phi_{\left(\theta_{1}, \theta_{2}, \theta_{3}\right)}(x)$ can be nonzero no matter how many discs $x$ are specified by $X$, so the calculation of the interaction function (24) can be very time consuming. Second, (23) does not seem to be of much relevance if we can observe $U_{X}$ but not $X$. This indicates that another kind of neighbor relation is needed when describing the Markov properties. Two other relations are therefore discussed below.

4.5.2. Local Markov property in terms of the dual graph. It is natural to ask whether $\boldsymbol{T}$-interaction processes in the sense of [2] are nearest-neighbor Markov point processes with respect to the neighbor relation defined by the dual graph. Below we show that this is not the case in general.

First consider the case of the quermass-interaction process. Applying the inclusionexclusion formulae given by the last identity in (13), (19), and (21), we obtain another representation of the quermass-interaction process density, namely as a product of terms corresponding to the cliques in the dual graph, excluding the following case of 3-cliques $\{i, j, k\} \in \mathscr{D}_{x}$ with $b_{i} \cap b_{j} \cap b_{k}=\varnothing$ :

$$
\begin{aligned}
f_{\left(\theta_{1}, \theta_{2}, \theta_{3}\right)}(x)= & \frac{1}{c_{\left(\theta_{1}, \theta_{2}, \theta_{3}\right)}} \prod_{i \in I_{1}(x)} \phi_{\left(\theta_{1}, \theta_{2}, \theta_{3}\right)}\left(x_{i}\right) \prod_{\{i, j\} \in I_{2}(x)} \phi_{\left(\theta_{1}, \theta_{2}, \theta_{3}\right)}\left(\left\{x_{i}, x_{j}\right\}\right) \\
& \times \prod_{\{i, j, k\} \in I_{3}(x)} \phi_{\left(\theta_{1}, \theta_{2}, \theta_{3}\right)}\left(\left\{x_{i}, x_{j}, x_{k}\right\}\right),
\end{aligned}
$$

where now

$$
\begin{gathered}
\phi_{\left(\theta_{1}, \theta_{2}, \theta_{3}\right)}\left(x_{i}\right)=\exp \left(\theta_{1} A\left(b_{i}\right)+\theta_{2} L\left(b_{i}\right)+\theta_{3}\right), \\
\phi_{\left(\theta_{1}, \theta_{2}, \theta_{3}\right)}\left(\left\{x_{i}, x_{j}\right\}\right)=\exp \left(-\theta_{1} A\left(b_{i} \cap b_{j}\right)-\theta_{2} L\left(b_{i} \cap b_{j}\right)-\theta_{3}\right), \\
\phi_{\left(\theta_{1}, \theta_{2}, \theta_{3}\right)}\left(\left\{x_{i}, x_{j}, x_{k}\right\}\right)=\exp \left(\theta_{1} A\left(b_{i} \cap b_{j} \cap b_{k}\right)+\theta_{2} L\left(b_{i} \cap b_{j} \cap b_{k}\right)+\theta_{3}\right) .
\end{gathered}
$$


This is of a somewhat similar form to the Hammersley-Clifford representation for a nearestneighbor Markov point process with respect to the neighbor relation defined by the dual graph; however, it is not exactly of the required form, since in (25) we do not have a product over all $u \in x$ but a product only over those $u$ generating nonempty cells in $\mathscr{B}_{x}$. More precisely, since it can be verified that this neighbor relation satisfies certain consistency conditions, the quermass-interaction process is not a nearest-neighbor Markov point process with respect to the dual graph (Theorem 4.13 of [2]).

Next, we do not expect the $N_{\mathrm{h}}$-interaction and $N_{\mathrm{ic}}$-interaction processes to be nearestneighbor Markov point processes with respect to the dual graph, since we have again been unable to obtain a Hammersley-Clifford representation.

In contrast, the $N_{\mathrm{bv}}$-interaction process is a nearest-neighbor Markov point process with respect to the dual graph, since the identity in (12) implies the Hammersley-Clifford representation,

$$
f_{\theta_{6}}(x)=\frac{1}{c_{\theta_{6}}} \prod_{\{i, j\} \in I_{2}(x)} \exp \left(2 \theta_{6}\right) \prod_{\{i, j, k\} \in I_{3}(x)} \exp \left(-3 \theta_{6}\right) .
$$

Note that (25) and (26) do not seem to be of much relevance if we can observe $\boldsymbol{U}_{X}$ but not $X$.

4.5.3. Local Markov property in terms of the connected components. In our opinion, the most relevant results are Propositions 4 and 5, below, where the first proposition states that $X$ is a connected component Markov point process [2], [4], [7], [30] and the second proposition specifies a spatial Markov property. As explained in further detail in [2], for a connected component Markov point process, the Papangelou conditional intensity depends only on local information with respect to the connected component relation ' $\sim_{x}$ ' defined as follows: for $u, v \in x, u \sim_{x} v$ if and only if $b(u)$ and $b(v)$ are contained in the same connected component $K$ of $u_{x}$. Thereby MCMC computations become 'local', as discussed further in Section 4.7. The spatial Markov property is discussed in Sections 4.5.4 and 4.5.5.

Proposition 4. The $\boldsymbol{T}$-interaction process with density (6) is a connected component Markov point process.

Proof. The density is of the form

$$
\frac{1}{c_{\boldsymbol{\theta}}} \prod_{K \in \mathcal{K}\left(u_{x}\right)} \exp \left(\theta_{1} A(K)+\theta_{2} L(K)+\theta_{3} \chi(K)+\theta_{4} N_{\mathrm{h}}(K)+\theta_{5} N_{\mathrm{ic}}(K)+\theta_{6} N_{\mathrm{bv}}(K)\right)
$$

where $\mathcal{K}\left(U_{x}\right)$ is the set of connected components of $u_{x}$. Thus, by Lemma 1 of [4], it is a connected component Markov point process.

In the discrete case (discs replaced by pixels) a Markov connected component field [33], which is also assumed to be a second-order Markov random field, has a density of a similar form as (27).

4.5.4. Spatial Markov property in terms of the overlap relation. Consider again the quermassinteraction process and, for the moment, assume that $R=\operatorname{supp}(Q)<\infty$. Let $W_{\ominus 2 R}=\{u \in$ $W: b(u, 2 R) \subseteq W\}$ be the $2 R$-clipped window of points in $W$ so that almost surely no disc of $X$ with center in $W_{\ominus 2 R}$ intersects another disc of $X$ with center in $W^{c}$, where $W^{c}=S \backslash W$. Split $X$ into $X^{(1)}, X^{(2)}$, and $X^{(3)}$ corresponding to discs with centers in $W_{\ominus 2 R}, W \backslash W_{\ominus 2 R}$, and $W^{c}$, respectively. The spatial Markov property [38] states that $X^{(1)}$ and $X^{(3)}$ are conditionally 


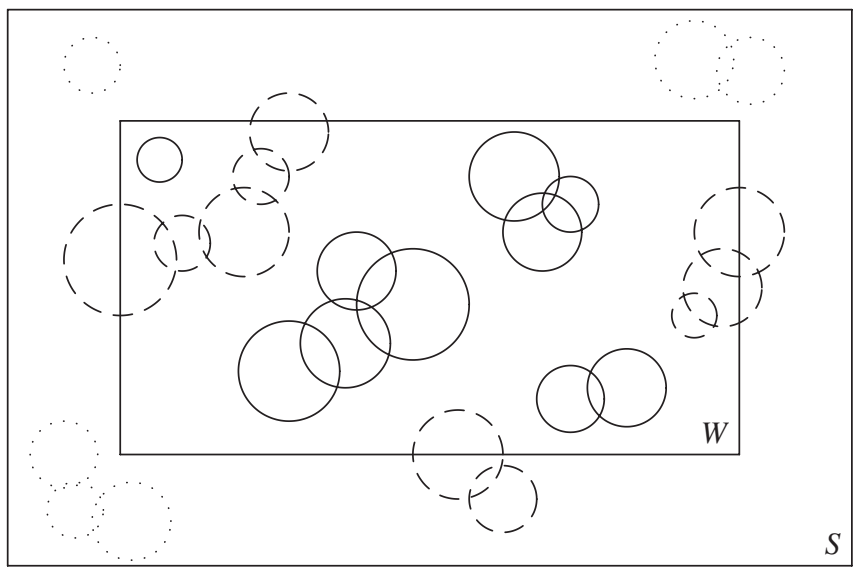

FIGURE 7: Illustrating possible realizations of $X^{(a)}$ (full circles), $X^{(b)}$ (dashed circles), and $X^{(c)}$ (dotted circles).

independent given $X^{(2)}$, and the conditional distribution $X^{(1)} \mid X^{(2)}=x^{(2)}$ has density

$$
\begin{aligned}
& f_{\theta_{1}, \theta_{2}, \theta_{3}}\left(x^{(1)} \mid x^{(2)}\right) \\
& \quad=\frac{1}{c_{\theta_{1}, \theta_{2}, \theta_{3}}\left(x^{(2)}\right)} \exp \left(\theta_{1} A\left(u_{x^{(1)} \cup x^{(2)}}\right)+\theta_{2} L\left(u_{x^{(1)} \cup x^{(2)}}\right)+\theta_{3} \chi\left(u_{x^{(1)} \cup x^{(2)}}\right)\right)
\end{aligned}
$$

with respect to the reference Poisson process $\Psi$ restricted to discs with centers in the $2 R$-clipped window. This is also a Markov point process with respect to the overlap relation restricted to $W_{\ominus 2 R}$, since the Papangelou conditional intensity $\lambda_{\theta}\left(x^{(1)}, v \mid x^{(2)}\right)$ corresponding to (28) is related to that in (8) by

$$
\lambda_{\theta}\left(x^{(1)}, v \mid x^{(2)}\right)=\lambda_{\theta}\left(x^{(1)} \cup x^{(2)}, v\right) .
$$

However, it is problematic to use this conditional process in practice, since both (28) and (29) depend on $U_{x^{(2)}} \backslash W$, which is not observable.

4.5.5. Spatial Markov property in terms of the connected component relation. The following spatial Markov property is more useful and applies for the general case of the $\boldsymbol{T}$-interaction process (6), using the fact that it is a connected component Markov point process (see also [18] and [30]). We split $X$ into $X^{(a)}, X^{(b)}$, and $X^{(c)}$ corresponding to discs belonging to connected components of $U_{X}$ which are respectively (a) contained in $W$, (b) intersecting both $W$ and $W^{c}$, and (c) contained in $W^{c}$; see Figure 7. Furthermore, let $x^{(b)}$ denote any feasible realization of $X^{(b)}$, i.e. $x^{(b)}$ is a finite configuration of discs such that $K$ intersects both $W$ and $W^{c}$ for all $K \in \mathcal{K}\left(U_{x^{(b)}}\right)$.

Proposition 5. Conditional on $X^{(b)}=x^{(b)}$, we have $X^{(a)}$ and $X^{(c)}$ are independent, and the conditional distribution of $X^{(a)}$ depends only on $x^{(b)}$ through $V=W \cap u_{x^{(b)}}$ and has density

$$
f_{\boldsymbol{\theta}}\left(x^{(a)} \mid V\right)=\frac{1}{c_{\boldsymbol{\theta}}(V)} \mathbf{1}\left[u_{x^{(a)}} \subseteq W \backslash V\right] \exp \left(\boldsymbol{\theta} \cdot \boldsymbol{T}\left(x^{(a)}\right)\right)
$$

with respect to the reference Poisson process of discs. 
Proof. Let $\Pi$ denote the distribution of $\Psi$ restricted to those finite configurations of discs with centers in $S$, and let $h_{\theta}$ denote the unnormalized density given by the exponential term in (27). Recall the 'Poisson expansion' (see, for example, [34])

$$
\begin{aligned}
\mathrm{P}(X \in F)= & \frac{1}{c_{\boldsymbol{\theta}}} \int_{F} h_{\boldsymbol{\theta}}(x) \Pi(\mathrm{d} x) \\
= & \frac{1}{c_{\boldsymbol{\theta}}} \exp \left(-\int_{S} \rho(u) \mathrm{d} u\right) \\
& \times \sum_{n=0}^{\infty} \frac{1}{n !} \int_{S} \int \cdots \int_{S} \int h_{\theta}(x) \mathbf{1}[x \in F] \rho\left(u_{1}\right) \mathrm{d} u_{1} Q\left(\mathrm{~d} r_{1}\right) \cdots \rho\left(u_{n}\right) \mathrm{d} u_{n} Q\left(\mathrm{~d} r_{n}\right)
\end{aligned}
$$

(where the term with $n=0$ is read as 1 if the empty configuration is in the event $F$ and 0 otherwise). From this and (27), we find that $\left(X^{(a)}, X^{(b)}, X^{(c)}\right)$ has joint density

$$
\begin{aligned}
f\left(x^{(a)}, x^{(b)}, x^{(c)}\right)= & \frac{1}{c_{\boldsymbol{\theta}}} \mathbf{1}\left[u_{x^{(a)}} \subseteq W \backslash u_{x^{(b)}}\right] h_{\boldsymbol{\theta}}\left(x^{(a)}\right) \mathbf{1}\left[u_{x^{(c)}} \subseteq W^{c} \backslash u_{x^{(b)}}\right] h_{\boldsymbol{\theta}}\left(x^{(c)}\right) \\
& \times \mathbf{1}\left[\text { for all } K \in \mathcal{K}\left(u_{x^{(b)}}\right): K \cap W \neq \varnothing, K \cap W^{c} \neq \varnothing\right] h_{\boldsymbol{\theta}}\left(x^{(b)}\right)
\end{aligned}
$$

with respect to the product measure $\exp \left(2 \int_{S} \rho(u) \mathrm{d} u\right) \Pi \times \Pi \times \Pi$. Thereby the proposition follows.

The density (30) may be useful for statistical applications, since it accounts for edge effects and depends only on the union of discs intersected by the observation window $W$. It is a hereditary density of a connected component Markov point process with discs contained in $W \backslash V$. Its Papangelou conditional intensity $\lambda_{\theta}\left(x^{(a)}, v \mid V\right)$ is simply given by

$$
\lambda_{\theta}\left(x^{(a)}, v \mid V\right)=\lambda_{\theta}\left(x^{(a)}, v\right) \mathbf{1}\left[u_{x^{(a)} \cup\{v\}} \subseteq W \backslash V\right] .
$$

\subsection{Stability}

Consider the 'unnormalized density' $h_{\boldsymbol{\theta}}(x)=\exp (\boldsymbol{\theta} \cdot \boldsymbol{T}(x))$ corresponding to the $\boldsymbol{T}$-interaction process with density $f_{\boldsymbol{\theta}}$ given in (6), and recall the definition of the parameter space $\Theta$, (5). In fact, we have not yet verified that $c_{\theta} \equiv \mathrm{E} h_{\theta}(\Psi \cap(S \times(0, \infty)))$ is finite for $\boldsymbol{\theta} \in \Theta$ and, hence, that $f_{\boldsymbol{\theta}}=h_{\boldsymbol{\theta}} / c_{\boldsymbol{\theta}}$ is a well-defined density with respect to the reference Poisson process $\Psi$ if $\theta \in \Theta$. In this subsection we discuss two stability properties which imply integrability of $h_{\theta}$ as well as other desirable properties.

4.6.1. Ruelle stability. This means that there exist positive constants $\alpha$ and $\beta$ such that $h_{\theta}(x) \leq$ $\alpha \beta^{n(x)}$ for all $x \in \mathcal{N}$ (in fact, this and other stability properties mentioned in this paper need only hold almost surely with respect to $\Psi$; however, for ease of presentation, we shall ignore such nullsets). Ruelle stability implies that $c_{\theta} \leq \alpha \exp \left((\beta-1) \int_{S} \rho(z) \mathrm{d} z\right)<\infty$, and we say that $f_{\theta}=h_{\theta} / c_{\theta}$ is a Ruelle stable density. Other implications of Ruelle stability are discussed in Section 2.1 of [22] and the references therein.

The main question addressed in [22] was how to establish Ruelle stability of the quermassinteraction process, and the following proposition provides a very easy proof of this issue in connection to the general case of the $\boldsymbol{T}$-interaction process (6) (since the proof is based on Lemma 2, the usefulness of the power tessellation is once again demonstrated).

Proposition 6. For all $\boldsymbol{\theta} \in \Theta, c_{\boldsymbol{\theta}}<\infty$ and $f_{\boldsymbol{\theta}}$ in (6) is a Ruelle stable density. If $\boldsymbol{\theta} \in \mathbb{R}^{6} \backslash \Theta$ then $c_{\theta}=\infty$. 
Proof. Note that a finite product of Ruelle stable functions is a Ruelle stable function. Let $\theta_{0}$ denote a real parameter. From Lemma 2 , it follows that $\chi, N_{\mathrm{h}}, N_{\mathrm{ic}}$, and $N_{\mathrm{bv}}$ are bounded above by $6 N$, so the functions $\exp \left(\theta_{0} W\right), W=\chi, N_{\mathrm{h}}, N_{\mathrm{ic}}, N_{\mathrm{bv}}$, are Ruelle stable for all $\theta_{0} \in \mathbb{R}$. Moreover, $\exp \left(\theta_{1} A+\theta_{2} L\right)$ is Ruelle stable if $a \equiv \int \exp \left(\pi \theta_{1} r^{2}+2 \pi \theta_{2} r\right) Q(\mathrm{~d} r)$ is finite, since $\exp \left(\theta_{1} A+\theta_{2} L\right) \leq \exp \left((a-1) \int_{S} \rho(z) \mathrm{d} z\right)$. On the other hand, the first term in the infinite sum in (2) is $a \exp \left(\theta_{3}+\theta_{5}\right) \int_{S} \rho(z) \mathrm{d} z$, where $\int_{S} \rho(z) \mathrm{d} z>0$; cf. Section 2.1. Consequently, $c_{\theta}=\infty$ if $a=\infty$.

4.6.2. Local stability. This means that there exists a constant $\beta$ such that, for all $x \in \mathcal{N}$ and all $v \in \Omega \backslash x$,

$$
\lambda_{\theta}(x, v) \leq \beta .
$$

This property is clearly implying Ruelle stability. Local stability is useful when establishing geometric ergodicity of MCMC algorithms (see [13], [34], and also Section 4.7), and it is needed in order to apply the dominating coupling from the past algorithm in [20] and [21] for making perfect simulations. Note that a finite product of locally stable functions is a locally stable function, since its Papangelou conditional intensity is given by a product of uniformly bounded Papangelou conditional intensities. The Papangelou conditional intensity (6) is a product of Papangelou conditional intensities corresponding to functions $h_{\theta_{0}}(x)=\exp \left(\theta_{0} W\left(\mathcal{U}_{x}\right)\right)$ with $W=A, L, \ldots$ and $\theta_{0}=\theta_{1}, \theta_{2}, \ldots$

As shown below, the picture of whether local stability is satisfied or not depends much on the particular type of model. In the following proposition, when we write 'in general', the proof of the proposition will show examples where local stability is not satisfied, depending on how $S$ and $\operatorname{supp}(Q)$ are specified, and it should be obvious to the reader that local stability will not be satisfied in many other cases as well. We let $\varepsilon=\inf \operatorname{supp}(Q)$ and $R=\sup \operatorname{supp}(Q)$.

Proposition 7. Local stability is satisfied for

(a) the A-interaction process if and only if $\theta_{1} \leq 0$ or $R<\infty$;

(b) the L-interaction process if $\theta_{2}=0$, or $R<\infty$ if $\theta_{2}>0$, or $\varepsilon>0$ and $R<\infty$ if $\theta_{2}<0$; otherwise in general it is not locally stable;

(c) the $\chi$-interaction process if $\theta_{3} \geq 0$, while in general it is not locally stable if $\theta_{3}<0$;

(d) the $N_{\mathrm{cc}}$-interaction process if $\theta_{3}=\theta_{4} \geq 0$ or both $\theta_{3}=\theta_{4}<0$ and $\varepsilon>0$, while it is not locally stable if $\theta_{3}=\theta_{4}<0$ and $\varepsilon=0$;

(e) the $N_{\mathrm{ic}}$-interaction process if $\theta_{5} \geq 0$ or $\varepsilon>0$, while it is not locally stable if $\theta_{5}<0$ and $\varepsilon=0$.

Moreover, local stability is in general not satisfied for

(f) the $N_{\mathrm{h}}$-interaction process unless $\theta_{4}=0$;

(g) the $N_{\mathrm{bv}}$-interaction process unless $\theta_{6}=0$.

Proof. Let $x \in \mathcal{N}$ and $v \in \Omega \backslash x$.

It follows from (11) that $\lambda_{\theta_{1}}(\varnothing, v)=\exp \left(\pi \theta_{1} r^{2}\right), \lambda_{\theta_{1}}(x, v) \leq \exp \left(\pi \theta_{1} r^{2}\right)$ if $\theta_{1} \geq 0$, and $\lambda_{\theta_{1}}(x, v) \leq 1$ if $\theta_{1} \leq 0$. Thereby part (a) follows, and in a similar way we verify part (b) in the case in which $\theta_{2} \geq 0$. It also follows from (11) that the $\chi$-interaction process is locally stable if $\theta_{3} \geq 0$. 


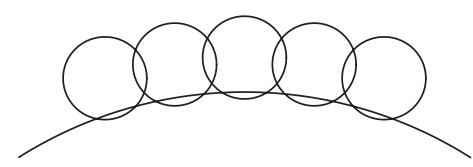

FIGURE 8: A configuration $x$ of $n=6$ discs intersected by another disc $b_{v}$ such that \#holes $\left(b_{v} \cap U_{x}\right)=$ $n-1=5$.

To verify part (b) in the case in which $\theta_{2}<0$, we first suppose that $\varepsilon>0$ and $R<\infty$, and use an argument which Wilfrid Kendall kindly pointed out to us. A boundary edge corresponding to an angle $0<\varphi<2 \pi$ and a disc of radius $r$ has length $\varphi r$, and it defines a sector of area $\varphi r^{2} / 2$. Since such sectors have disjoint interiors,

$$
A\left(u_{x}\right) \geq \sum_{j} \frac{\varphi_{j} r_{j}^{2}}{2} \geq \frac{\varepsilon^{2}}{2} \sum_{j} \varphi_{j},
$$

where the sum is over all boundary edges. Hence,

$$
L\left(u_{x}\right)=\sum_{j} \varphi_{j} r_{j} \leq R \sum_{j} \varphi_{j} \leq \frac{2 R}{\varepsilon^{2}} A\left(u_{x}\right)<c,
$$

where $c$ is a finite constant (since the discs specified by $x$ have centers in the bounded region $S$ and their radii are bounded by $R, A\left(U_{x}\right)$ has an upper bound). Consequently,

$$
L(x, v)=L\left(b_{v}\right)-L\left(b_{v} \cap u_{x}\right) \geq 2 \pi \varepsilon-c,
$$

and so local stability is established when $\theta_{2}<0, \varepsilon>0$, and $R<\infty$.

On the other hand, suppose that $\varepsilon=0$ or $R=\infty$. Let $r$ denote the radius of $b_{v}$, let $0<\delta<r$, and consider the infinite configuration of discs of radii $\delta$ and centers at the sites of an equilateral triangular lattice of side length $2 \delta$. The proportion of $\mathbb{R}^{2}$ covered by these discs is the so-called maximal packing degree $p=\pi / \sqrt{12}$ (a number independent on how $\delta$ is chosen). Now, suppose that $x$ is the subconfiguration of all such discs contained in $b_{v}$. As either $\delta$ decreases to 0 or $r$ increases to $\infty, n(x) \delta^{2} / r^{2}$ converges to $p$, and so

$$
L(x, v)=L\left(b_{v}\right)-L\left(b_{v} \cap U_{x}\right)=2 \pi r-2 \pi \delta n(x)
$$

is converging to $-\infty$. Hence, if $\theta_{2}<0$, the local stability condition is violated, and so part (b) is verified.

To show an example where the $\chi$-interaction process is not locally stable if $\theta_{3}<0$, consider Figure 8. Suppose that $x=\left\{x_{1}, \ldots, x_{n}\right\}$ corresponds to the pairwise overlapping small discs in Figure 8 and that $b_{v}$ corresponds to the large disc. Then each pair $x_{i}, x_{i+1}$ together with $b_{v}$ form one hole, and $N_{\mathrm{h}}\left(b_{v} \cap \mathcal{U}_{x}\right)=n-1$. Since $n$ may be arbitrarily large, using (11) again, we obtain part (c). Note that $b_{v}$ does not need to be so large compared to the other discs in Figure 8; it is only chosen in this way for illustrative purposes. For example, all the discs may be of a very similar size so that $N_{\mathrm{h}}\left(b_{v} \cap U_{x}\right)=n-1$ still (then the discs in $x$ will be much more overlapping than indicated in Figure 8). More precisely, whether this holds or not depends on how large $S$ is compared to $\operatorname{supp}(Q)$. For instance, if $S$ is a disc with radius $R$ and 
$\Omega=S \times\{2 R\}$ then $\chi\left(U_{x}\right)=1$ for all $x \in \mathcal{N}$, and so the $\chi$-interaction process is locally stable for all $\theta_{3} \in \mathbb{R}$.

For part (d), we use the fact that

$$
N_{\mathrm{cc}}(x, v)=1-\#\left\{\text { connected components in } \mathcal{U}_{x} \text { which are intersected by } b_{v}\right\} .
$$

Hence, we immediately obtain local stability if $\theta_{3}=\theta_{4} \geq 0$. Suppose instead that $\theta_{3}=\theta_{4}<0$. By (33), $N_{\mathrm{cc}}(x, v)$ has no lower bound if $\varepsilon=0$, since the discs in $x$ can be disjoint and still all intersect $b_{v}$. On the other hand, if $\varepsilon>0$ then $1-N_{\mathrm{cc}}(x, v)$ is at most equal to the maximal number of disjoint discs with radius $\varepsilon$ and centers in $S$. Thereby part (d) is verified. The proof of part (e) is similar, using instead the fact that

$$
N_{\text {ic }}(x, v)=\mathbf{1}_{\text {ic }}(x, v)-\#\left\{\text { isolated cells in } U_{x} \text { which are contained in } b_{v}\right\},
$$

where $\mathbf{1}_{\text {ic }}(x, v)$ is the indicator function which is 1 if $B_{v}$ is an isolated cell in $\mathscr{B}_{x \cup\{v\}}$ and 0 otherwise.

The $N_{\mathrm{h}}$-interaction process with $\theta_{4}=0$ and the $N_{\mathrm{bv}}$-interaction process with $\theta_{6}=0$ are nothing but the Poisson process $\Psi$, and so local stability is obviously satisfied. By similar arguments as above in the proof of part (c), when $\theta_{3}<0$, there are in general no uniform upper and lower bounds on either $N_{\mathrm{h}}(x, v)$ or $N_{\mathrm{bv}}(x, v)$. Thereby, parts (f) and (g) follow.

Proposition 7 immediately extends to the conditional quermass-interaction process with density (28) and the conditional $\boldsymbol{T}$-interaction process in (30). Note that if the indicator term in (30) is 1 , it implies that the radius of any disc in $x^{(a)}$ is less than a constant. Consequently, (a) the conditional $A$-interaction process given by (30) is always locally stable, and (b) the $L$-interaction process given by (30) is locally stable if either $\theta_{2} \geq 0$ or $\theta_{2}<0$ and $\varepsilon>0$, and in general it is not locally stable if $\theta_{2}<0$ and $\varepsilon=0$.

\subsection{MCMC algorithms}

For simulation of the $\boldsymbol{T}$-interaction process (6), the conditional quermass-interaction point process with density (28), or the conditional $\boldsymbol{T}$-interaction process with density (30), we use a simple version of the birth-death type Metropolis-Hastings algorithm studied in [13], [14], and [34]. For specificity, we first consider the $\boldsymbol{T}$-interaction process $X$ with Papangelou conditional intensity $\lambda_{\theta}(x, v)$ given by (8).

In the Metropolis-Hastings algorithm if $x$ is the state at iteration $t$, we generate a proposal which is either a 'birth' $x \cup\{v\}$ of a new disc $v=(z, r)$ or a 'death' $x \backslash\left\{x_{i}\right\}$ of an old disc $x_{i} \in x$. Each kind of proposal may happen with equal probability $\frac{1}{2}$. Define

$$
r_{\boldsymbol{\theta}}(x, v)=\lambda_{\boldsymbol{\theta}}(x, v) \frac{\int_{S} \rho(s) \mathrm{d} s}{\rho(z)(n(x)+1)} .
$$

In the case of a birth proposal, $v$ follows the normalized intensity measure of $\Psi$, i.e. $z$ and $r$ are independent, $z$ has a density on $S$ proportional to $\rho$, and $r$ follows the mark distribution $Q$. This proposal is accepted as the state at iteration $t+1$ with probability $\min \left\{1, H_{\theta}(x, v)\right\}$, where the Hastings ratio is given by $H_{\theta}(x, v)=r_{\theta}(x, v)$. In the case of a death proposal, $x_{i}$ is a uniformly selected point from $x$, and the Hastings ratio in the acceptance probability of the proposal is now given by $H_{\theta}\left(x, x_{i}\right)=1 / r_{\theta}\left(x \backslash\left\{x_{i}\right\}, x_{i}\right)$ (in the special case where $x=\varnothing$, we do nothing). Finally, if neither kind of proposal is accepted, we retain $x$ at iteration $t+1$.

As verified in [14], the generated Markov chain is aperiodic and positive Harris recurrent, the chain converges towards the distribution of $X$, and Birkhoff's ergodic theorem establishes 
convergence of Monte Carlo estimates of mean values with respect to (6). If local stability is satisfied (see Proposition 7), the chain is geometrical ergodic and, hence, a central limit theorem applies for Monte Carlo estimates [6], [34], [39]. Moreover, from a computational perspective, the important point of the algorithm is that it only involves calculating the Papangelou conditional intensity, so only local computations of the statistics appearing in (8) are needed; cf. Sections 4.3-4.5.

In theory we may use any state of $\mathcal{N}$ as the initial state of the algorithm, but we have mainly used three kinds of initial states:

(i) the extreme case of the empty configuration $\varnothing$;

(ii) if local stability is satisfied, the other extreme case is given by a realization from a Poisson process $\Xi$ with intensity measure $\beta \rho(z) \mathrm{d} z Q(\mathrm{~d} r)$, where $\beta$ is the upper bound in (32);

(iii) a realization of the reference Poisson process $\Psi$ (an intermediate case of (i) and (ii) if $\beta>1)$.

In fact, local stability ensures that the Poisson process in (ii) can be coupled with $X$ so that $X \subseteq \Xi$, and this kind of domination can be exploited to make perfect simulations of $X$, using a dominating coupling from the past algorithm [19], [21].

The algorithm for simulating from the conditional processes with densities (28) and (30) is the same except that we replace $\lambda_{\theta}(x, v)$ in (34) by the Papangelou conditional intensities in (29)-(31), and that the state space has of course to be in accordance with (28) and (30). The convergence properties and computations are therefore similar to those discussed above. The initial states are of course slightly different, where we modify the Poisson process in (ii) or (iii) above as follows. For (28), we restrict the Poisson process in (ii) or (iii) so that the centers are in $W_{\ominus 2 R}$. For (30), we first restrict the Poisson process in (ii) or (iii) so that the centers are in $W$, and second, when we make a simulation from this Poisson process, we finally omit those discs which are not included in $W \backslash V$.

\section{Extensions and open problems}

We conclude with some remarks on possible extensions of this work and on some open problems.

We demonstrated the usefulness of the power tessellation in connection to the $\boldsymbol{T}$-interaction process (4), and argued why this model is best viewed as a connected component Markov point process. For the specification of the sufficient statistic $\boldsymbol{T}$, other geometric characteristics than those in (4) may be of interest to include, for example, the shape characteristic for the connected components $K$ such as $A(K) / L(K)^{2}$. The power tessellation will also be a useful tool for such extensions, not least since local calculations can be carried out as discussed in Section 4.4.

We confined ourselves to the case of discs in $\mathbb{R}^{2}$, though many concepts and results can be extended to the general case of balls in $\mathbb{R}^{d}$. The planar case, $d=2$, is already complicated enough, and indeed the power tessellation in higher dimensions becomes more complicated; cf. [10]. The planar case is of principal importance for applications in spatial statistics and stochastic geometry (see, e.g. [8] and [41]), and the spatial case, $d=3$, is of particular importance in physics and computational biology (see, e.g. [11], [24], [25], and [26]).

The $\boldsymbol{T}$-interaction processes obviously provide a large and flexible class of random models for unions of discs. It would be interesting to get a better understanding of the importance of the parameters $\theta_{1}, \ldots, \theta_{6}$; cf. Section 4.2. For instance, how different are the models which have been simulated in Section 4.2 and how different would a fitted $L$-interaction process be if 
the true model is an $A$-interaction process? Probably, to answer such questions, an extensive simulation study will be required.

As noted in Section 4.6, the dominating coupling from the past algorithm [20], [21] for making perfect simulations requires local stability. Moreover, to make this algorithm work in practice, some monotonicity property like (9) or an antimonotonicity property like (10) is useful, but apart from the $A$-interaction process, our models are in general neither attractive nor repulsive; cf. Proposition 2. How difficult is it to make a perfect simulation of, for example, the $L$-interaction process?

Also, extensions of our $\boldsymbol{T}$-interaction models to infinite configurations of discs would be of interest, particularly for applications in statistical physics. Such extensions are possible for quermass-interaction models, at least if $Q$ has bounded support (see [22]), but how do we extend the other kind of $\boldsymbol{T}$-interaction models? The usual approach is to use a local specification in the sense of [37] or, equivalently, to specify the Papangelou conditional intensity for the infinite process [12], [35], but this would require that the connected components are almost surely bounded. See the somewhat related discussion in [33] concerning infinite extensions of Markov connected component fields.

A problem related to infinite extensions of $\boldsymbol{T}$-interaction models is the issue of phase transition. The $A$-interaction model exhibits phase transition, at least if the radii are all fixed at a constant value [15], [40], but what about other $\boldsymbol{T}$-interaction models?

Finally, we are currently exploiting the results in this paper to study the statistical aspects, in particular likelihood-based inference, in a follow up paper [32].

\section{Appendix A. Successive construction of power tessellations}

In this appendix we explain how to construct a new power tessellation of a union of discs by adding a new disc (Section A.1) or deleting an old disc (Section A.2), assuming that the old power tessellation is known. The constructions can easily be extended to keep track on the connected components of the union of discs, but to save space we omit these details.

\section{A.1. The case where a new disc is added}

Suppose that we want to construct a new power tessellation $\mathscr{B}^{\text {new }}$ of a union $\mathcal{U}^{\text {new }}=\bigcup_{1}^{n} b_{i}$ of $n \geq 1$ discs in general position, where we are adding the disc $b_{n}$ and we have already constructed the power tessellation $\mathscr{B}^{\text {old }}$ of $U^{\text {old }}=\bigcup_{1}^{n-1} b_{i}$ based on the $n-1$ other discs (if $n=1$ then $\mathcal{B}^{\text {old }}$ and $\mathcal{U}^{\text {old }}$ are empty). More precisely, with respect to $\mathcal{B}^{\text {old }}$, we assume that we know all the old edges. We denote the old interior edges by $\left[u_{i, j}^{\text {old }}, v_{i, j}^{\text {old }}\right]$ and the old boundary edges by $\left\lfloor u_{i}^{\text {old }}, v_{i}^{\text {old }}\right\rceil$ or $\partial b_{i}^{\text {old }}$. We want to construct the new tessellation $\mathscr{B}^{\text {new }}$ of $\mathcal{U}^{\text {new }}=\mathcal{U}^{\text {old }} \cup b_{n}$ by finding its interior edges $\left[u_{i, n}^{\text {new }}, v_{i, n}^{\text {new }}\right]$ and boundary edges $\left\lfloor u_{n}^{\text {new }}, v_{n}^{\text {new }}\right\rceil$ associated to the new cell $B_{n}^{\text {new }}$. This is done in steps (ii) and (iv), below. Moreover, to obtain the remaining new edges, we modify old interior edges $\left[u_{i, j}^{\text {old }}, v_{i, j}^{\text {old }}\right]$ and old boundary edges $\left\lfloor u_{i}^{\text {old }}, v_{i}^{\text {old }}\right\rceil$ or $\partial b_{i}^{\text {old }}$, noting that a 'modified old edge' can be unchanged, reduced, or disappearing. This is done in steps (iii) and (v), below. Note that steps (i), (ii), and (iv) determine the new cells, i.e. which of the sets $B_{1}^{\text {new }}, \ldots, B_{n}^{\text {new }}$ are empty or not.

(i) Considering old discs intersecting the new disc. If $b_{n}$ is contained in some disc $b_{j}$ with $j<n$ then $B_{n}^{\text {new }}$ is empty and so $\mathscr{B}^{\text {new }}=\mathscr{B}^{\text {old }}$ is unchanged. Assume that $b_{n}$ is not contained in any disc $b_{j}$ with $j<n$ and, without loss of generality, that $b_{n}$ intersects $B_{1}^{\text {old }}, \ldots, B_{i}^{\text {old }}$ but not $B_{i+1}^{\text {old }}, \ldots, B_{n-1}^{\text {old }}$, where $0 \leq i \leq n-1$ (setting $i=0$ if $b_{n}$ has no intersection). Then 
$B_{j}^{\text {new }}=B_{j}^{\text {old }}$ is unchanged for $j=i+1, \ldots, n-1$, so it suffices below to find the edges of $B_{1}^{\text {new }}, \ldots, B_{i}^{\text {new }}$ and $B_{n}^{\text {new }}$.

If $i=0$ then $B_{n}^{\text {new }}=b_{n}$ is an isolated cell with boundary edge $\partial b_{n}$. In steps (ii)-(v) we assume that $i \geq 1$.

(ii) Finding the interior edges of $B_{n}^{\text {new }}$. To obtain the interior edges of $B_{n}^{\text {new }}$, for $j=1, \ldots, i$, we start by assigning $e_{j, n}^{\text {new }} \leftarrow\left[u_{j, n}^{\text {new }}, v_{j, n}^{\text {new }}\right]$, considering $u_{j, n}^{\text {new }}$ and $v_{j, n}^{\text {new }}$ as (potential) boundary vertices given by the endpoints of the chord $E_{j, n}$. Furthermore, for $k=1, \ldots, i$ with $k \neq j$, if $e_{j, n}^{\text {new }} \cap H_{n, k}=\varnothing$ (or, equivalently, $u_{j, n}^{\text {new }} \notin H_{n, k}$ and $v_{j, n}^{\text {new }} \notin H_{n, k}$, since $H_{n, k}$ is convex), we obtain $e_{j, n}^{\text {new }} \leftarrow \varnothing$ and we can stop the $k$-loop, else $e_{j, n}^{\text {new }} \leftarrow e_{j, n}^{\text {new }} \cap H_{n, k}$. In the latter case either both vertices are contained in $H_{n, k}$ and so the edge remains unchanged, or exactly one vertex is not contained in $H_{n, k}$, e.g. $u_{j, n}^{\text {new }} \notin H_{n, k}$ but $v_{j, n}^{\text {new }} \in H_{n, k}$, in which case $u_{j, n}^{\text {new }}$ becomes an interior vertex given by the point $e_{j, n}^{\text {new }} \cap \partial H_{n, k}$ while $v_{j, n}^{\text {new }}$ is unchanged. In this way we find all the interior edges of $B_{n}^{\text {new }}$, and all the interior and boundary vertices of $B_{n}^{\text {new }}$.

Since we have assumed that $i>0, B_{n}^{\text {new }}$ is empty if and only if it has no interior edges.

(iii) Modifying the old interior edges. At the same time as we do step (ii) above, we also check whether each interior edge $e_{j, k}^{\text {old }}=\left[u_{j, k}^{\text {old }}, v_{j, k}^{\text {old }}\right]$ of $\mathscr{B}^{\text {old }}$ with $j<k \leq i$ should be kept, reduced, or omitted when we consider $\mathscr{B}^{\text {new }}$ (recalling that $e_{j, k}^{\text {new }}=e_{j, k}^{\text {old }}$ is unchanged if $j>i$ or $k>i$ ). We have

$$
e_{j, k}^{\text {new }}=e_{j, k}^{\text {old }} \cap H_{j, n}=e_{j, k}^{\text {old }} \cap H_{k, n} .
$$

Thus, $e_{j, k}^{\text {new }}$ is empty if $u_{j, k}^{\text {old }} \notin H_{k, n}$ and $v_{j, k}^{\text {old }} \notin H_{k, n}$, while $e_{j, k}^{\text {new }}=e_{j, k}^{\text {old }}$ if $u_{j, k}^{\text {old }} \in H_{k, n}$ and $v_{j, k}^{\text {old }} \in H_{k, n}$. Furthermore, if $u_{j, k}^{\text {old }} \in H_{k, n}$ and $v_{j, k}^{\text {old }} \notin H_{k, n}$ then $e_{j, k}^{\text {new }} \stackrel{j, k}{=}\left[u_{j, k}^{\text {old }}, v_{j, k}^{j, k}\right]$, where $v_{j, k}^{\text {new }}$ is the point given by $e_{j, k}^{\text {old }} \cap \partial H_{k, n}$. Similarly, if $u_{j, k}^{\text {old }} \notin H_{k, n}$ and $v_{j, k}^{\text {old }} \in H_{k, n}$ then $e_{j, k}^{\text {new }}=$ $\left[u_{j, k}^{\text {new }}, v_{j, k}^{\text {old }}\right]$, where $u_{j, k}^{\text {new }}$ is the point given by $e_{j, k}^{\text {old }} \cap \partial H_{k, n}$.

Note that, for each $j \leq i, B_{j}^{\text {new }}$ is empty if and only if it has no interior edge.

(iv) Finding the boundary edges of $B_{n}^{\text {new }}$. Suppose that $B_{n}^{\text {new }}$ has $m>0$ boundary vertices $w_{1}^{\text {new }}, \ldots, w_{m}^{\text {new }}$. Note that $m$ is an even number, and we can organize the boundary vertices such that $w_{1}^{\text {new }}=z_{n}+r_{n}\left(\cos \varphi_{1}^{\text {new }}, \sin \varphi_{1}^{\text {new }}\right), \ldots, w_{m}^{\text {new }}=z_{n}+r_{n}\left(\cos \varphi_{m}^{\text {new }}, \sin \varphi_{m}^{\text {new }}\right)$, where $0 \leq \varphi_{1}^{\text {new }}<\cdots<\varphi_{m}^{\text {new }}<2 \pi$. Then $B_{n}^{\text {new }}$ has $m / 2$ boundary edges, namely

$$
\left\lfloor w_{2}^{\text {new }}, w_{3}^{\text {new }}\right\rceil,\left\lfloor w_{4}^{\text {new }}, w_{5}^{\text {new }}\right\rceil, \ldots,\left\lfloor w_{m}^{\text {new }}, w_{1}^{\text {new }}\right\rceil \quad \text { if } z_{n}+\left(r_{n}, 0\right) \in H_{n, j} \text { for all } j=1, \ldots, i
$$

and

$$
\left\lfloor w_{1}^{\text {new }}, w_{2}^{\text {new }}\right\rceil,\left\lfloor w_{3}^{\text {new }}, w_{4}^{\text {new }}\right\rceil, \ldots,\left\lfloor w_{m-1}^{\text {new }}, w_{m}^{\text {new }}\right\rceil \text { otherwise. }
$$

(v) Modifying the old boundary edges. Finally, we modify the boundary edges $\left\lfloor u_{j}^{\text {old }}, v_{j}^{\text {old }}\right\rceil$ of $\mathscr{B}^{\text {old }}$ considering $\mathscr{B}^{\text {new }}$ and $j \leq i$ (noting that $\left\lfloor u_{j}^{\text {old }}, v_{j}^{\text {old }}\right\rceil$ is a boundary edge of $\mathscr{B}^{\text {new }}$ too if $j>i$ ). This is done in a similar way as in step (iv). Suppose that $B_{j}^{\text {new }}$ has $m_{j}>0$ boundary vertices $w_{1}^{\text {new }}, \ldots, w_{m_{j}}^{\text {new }}$, which we organize as in step (iv). Then $B_{j}^{\text {new }}$ has boundary edges

$$
\begin{aligned}
\left\lfloor w_{2}^{\text {new }}, w_{3}^{\text {new }}\right\rceil,\left\lfloor w_{4}^{\text {new }},\right. & \left.w_{5}^{\text {new }}\right\rceil, \ldots,\left\lfloor w_{m_{j}}^{\text {new }}, w_{1}^{\text {new }}\right\rceil \\
& \text { if } z_{j}+\left(r_{j}, 0\right) \in H_{j, k} \text { for all } k \leq n \text { with } k \neq j \text { and } b_{j} \cap b_{k} \neq \varnothing
\end{aligned}
$$

and

$$
\left\lfloor w_{1}^{\text {new }}, w_{2}^{\text {new }}\right\rceil,\left\lfloor w_{3}^{\text {new }}, w_{4}^{\text {new }}\right\rceil, \ldots,\left\lfloor w_{m_{j-1}}^{\text {new }}, w_{m_{j}}^{\text {new }}\right\rceil \text { otherwise. }
$$




\section{A.2. The case where a disc is deleted}

Suppose that we are deleting the disc $b_{n}$ from a configuration $\left\{b_{1}, \ldots, b_{n}\right\}$ of $n \geq 1$ discs, which are assumed to be in general position. We also assume that we know the power tessellation $\mathscr{B}^{\text {old }}$ of $\mathcal{U}^{\text {old }}=\bigcup_{1}^{n} b_{i}$. Below we explain how to construct the new power tessellation $\mathscr{B}^{\text {new }}$ of $\mathcal{U}^{\text {new }}=\bigcup_{1}^{n-1} b_{i}$. More precisely, with respect to $\mathscr{B}^{\text {old }}$, we assume that we know all the interior edges $\left[u_{i, j}^{\text {old }}, v_{i, j}^{\text {old }}\right]$ and all the boundary edges $\left\lfloor u_{i}^{\text {old }}, v_{i}^{\text {old }}\right\rceil$. We want to construct the tessellation $\mathcal{B}^{\text {new }}$ of $U^{\text {new }}=u^{\text {old }} \backslash b_{n}$ by finding the interior edges $\left[u_{i, j}^{\text {new }}, v_{i, j}^{\text {new }}\right]$ and the boundary edges $\left\lfloor u_{i}^{\text {new }}, v_{i}^{\text {new }}\right\rceil$ associated to each new cell $B_{i}^{\text {new }}$, noting that $B_{i}^{\text {new }}$ either agrees with $B_{i}^{\text {old }}$ or is an enlargement of $B_{i}^{\text {old }}$ or is a completely new cell. One possibility could be to 'reverse' the construction in Section A.1, where a new disc is added; however, we realized that it is easier to create the new edges without reversing the construction in Section A.1 but using a construction as described below. This is partly explained by the fact that an old empty set $B_{i}^{\text {old }}$ may possibly be replaced by a nonempty set $B_{i}^{\text {new }}$.

(i) Considering the discs intersecting the disc which is deleted. Clearly, if $B_{n}^{\text {old }}$ is empty then $\mathscr{B}^{\text {new }}=\mathscr{B}^{\text {old }}$ is unchanged. Assume that $B_{n}^{\text {old }}$ is a nonempty cell and, without loss of generality, that $b_{n}$ intersects $b_{1}, \ldots, b_{i}$ but not $b_{i+1}, \ldots, b_{n-1}$, where $0 \leq i \leq n-1$ (setting $i=0$ if $b_{n}$ has no intersection). Then it suffices to find the edges of $B_{1}^{\text {new }}, \ldots, B_{i}^{\text {new }}$, since $B_{j}^{\text {new }}=B_{j}^{\text {old }}$ is unchanged for $j=i+1, \ldots, n-1$. If $i=0$ then $B_{n}^{\text {old }}=b_{n}$ is an isolated cell, and so $B_{1}^{\text {new }}=B_{1}^{\text {old }}, \ldots, B_{n-1}^{\text {new }}=B_{n-1}^{\text {old }}$ are unchanged. In the following steps (ii)-(iv), suppose that $i>0$.

(ii) Finding the new interior edges. If $i=1$, no new interior edge appears. Suppose that $i \geq 2$. We want to determine each set $e_{j, k}^{\text {new }}$ with $j<k \leq i$. We start by assigning all cells $B_{1}^{\text {new }}, \ldots, B_{i}^{\text {new }}$ to be nonempty and by assigning $e_{j, k}^{\text {new }} \leftarrow\left[u_{j, k}^{\text {new }}, v_{j, k}^{\text {new }}\right]$, considering $u_{j, k}^{\text {new }}$ and $v_{j, k}^{\text {new }}$ as (potential) boundary vertices given by the endpoints of the chord $E_{j, k}$. Consider a loop with $l=1, \ldots, i$ and $l \neq j, k$. If $e_{j, k}^{\text {new }} \cap H_{k, l}=\varnothing$ (or, equivalently, $u_{j, k}^{\text {new }} \notin H_{k, l}$ and $v_{j, k}^{\text {new }} \notin H_{k, l}$, since $H_{k, l}$ is convex), we find that $e_{j, k}^{\text {new }}$ is empty and we can stop the $l$-loop. Otherwise, assign $e_{j, k}^{\text {new }} \leftarrow e_{j, k}^{\text {new }} \cap H_{k, l}$, where we note that only the following two cases can occur. First, if both vertices of $e_{j, k}^{\text {new }}$ are contained in $H_{k, l}$, the edge remains unchanged. Second, if exactly one vertex is not contained in $H_{k, l}$, e.g. $u_{j, k}^{\text {new }} \notin H_{k, l}$ but $v_{j, k}^{\text {new }} \in H_{k, l}$, then $u_{j, k}^{\text {new }}$ becomes an interior vertex given by the point $e_{j, k}^{\text {new }} \cap \partial H_{k, l}$ while $v_{j, k}^{\text {new }}$ is unchanged. When the loop is finished, we have determined all the new interior edges, including the information whether their endpoints are interior or boundary vertices.

(iii) Determining the new cells. For each $j \leq i$, we determine if $B_{j}^{\text {new }}$ is a new cell by checking if it has an edge. Suppose that $B_{j}^{\text {new }}$ has no interior edge, i.e. it is either an empty set or a new isolated cell. If an arbitrary fixed point of $b_{j}$ is included in $H_{j, l}$ for all $l=1, \ldots, n-1$ with $l \neq j$ then $B_{j}$ has exactly one boundary edge and it is an isolated cell. Otherwise, $B_{j}^{\text {new }}$ is empty. In this way we determine whether each $B_{j}^{\text {new }}$ is empty or a new cell, including whether it is an isolated cell.

(iv) Finding the new boundary edges. We have already determined the new isolated boundary edges in step (iii). Consider a nonisolated cell $B_{j}^{\text {new }}$ with $j \leq i$ with boundary vertices $w_{k}^{\text {new }}=z_{j}+r_{j}\left(\cos \varphi_{k}^{\text {new }}, \sin \varphi_{k}^{\text {new }}\right), k=1, \ldots, m_{j}$. Recall that $m_{j}>0$ is an even number and we organize the vertices so that $0 \leq \varphi_{1}^{\text {new }}<\cdots<\varphi_{m_{j}}^{\text {new }}<2 \pi$; cf. step (iv) in Section A.1. Then $B_{j}^{\text {new }}$ has $m_{j} / 2$ boundary edges, namely

$$
\left\lfloor w_{2}^{\text {new }}, w_{3}^{\text {new }}\right\rceil,\left\lfloor w_{4}^{\text {new }}, w_{5}^{\text {new }}\right\rceil, \ldots,\left\lfloor w_{m_{j}}^{\text {new }}, w_{1}^{\text {new }}\right\rceil \quad \text { if } z_{j}+\left(r_{j}, 0\right) \in H_{j, l} \text { for all } l=1, \ldots, i
$$


and

$$
\left\lfloor w_{1}^{\text {new }}, w_{2}^{\text {new }}\right\rceil,\left\lfloor w_{3}^{\text {new }}, w_{4}^{\text {new }}\right\rceil, \ldots,\left\lfloor w_{m_{j}-1}^{\text {new }}, w_{m_{j}}^{\text {new }}\right\rceil \text { otherwise }
$$

\section{Acknowledgements}

We are grateful to Lars Døvling Andersen, Herbert Edelsbrunner, and Wilfrid Kendall for useful comments. Supported by the Danish Natural Science Research Council, grant 272-060442, 'Point process modelling and statistical inference', and by grants GAČR 201/06/0302 and GAČR 201/05/H007.

\section{References}

[1] Aurenhammer, F. (1987). Power diagrams: properties, algorithms and applications. SIAM J. Comput. 16, 78-96.

[2] Baddeley, A. AND Møller, J. (1989). Nearest-neighbour Markov point processes and random sets. Internat. Statist. Rev. 2, 89-121.

[3] Baddeley, A. J. and van Lieshout, M. N. M. (1995). Area-interaction point processes. Ann. Inst. Statist. Math. 46, 601-619.

[4] Baddeley, A. J., van Lieshout, M. N. M. and Møller, J. (1996). Markov properties of cluster processes. Adv. Appl. Prob. 28, 346-355.

[5] Barndorff-Nielsen, O. E. (1978). Information and Exponential Families in Statistical Theory. John Wiley, Chichester.

[6] Chan, K. S. and Geyer, C. J. (1994). Discussion of the paper 'Markov chains for exploring posterior distributions' by Luke Tierney. Ann. Statist. 22, 1747.

[7] Chin, Y. C. And Baddeley, A. J. (2000). Markov interacting component processes. Adv. Appl. Prob. 32, 597619.

[8] Cressie, N. A. C. (1993). Statistics for Spatial Data, 2nd edn. John Wiley, New York.

[9] Diggle, P. (1981). Binary mosaics and the spatial pattern of heather. Biometrics 37, 531-539.

[10] Edelsbrunner, H. (1995). The union of balls and its dual shape. Discrete Computational Geometry 13, 415440.

[11] Edelsbrunner, H. (2004). Biological applications of computational topology. In Handbook of Discrete and Computational Geometry. eds J. Goodman and J. O’Rourke, Chapman \& Hall/CRC, Boca Raton, FL, pp. 13951412.

[12] Georgir, H.-O. (1976). Canonical and grand canonical Gibbs states for continuum systems. Commun. Math. Phys. 48, 31-51.

[13] Geyer, C. J. (1999). Likelihood inference for spatial point processes. In Stochastic Geometry: Likelihood and Computation, eds O. E. Barndorff-Nielsen et al., Chapman \& Hall/CRC, Boca Raton, FL, pp. 79-140.

[14] Geyer, C. J. AND MøLler, J. (1994). Simulation procedures and likelihood inference for spatial point processes. Scand. J. Statist. 21, 359-373.

[15] HäGgström, O., van Lieshout, M. N. M. AND MøLler, J. (1999). Characterization results and Markov chain Monte Carlo algorithms including exact simulation for some spatial point processes. Bernoulli 5, 641-659.

[16] Hall, P. (1988). Introduction to the Theory of Coverage Processes. John Wiley, New York.

[17] Hanisch, K.-H. (1981). On classes of random sets and point processes. Serdica 7, 160-167.

[18] Kendall, W. S. (1990). A spatial Markov property for nearest-neighbour Markov point processes. J. Appl. Prob. 28, 767-778.

[19] Kendall, W. S. (1998). Perfect simulation for the area-interaction point process. In Probability Towards 2000 (Springer Lecture Notes Statist. 128), eds L. Accardi and C. Heyde, Springer, New York, pp. 218-234.

[20] Kendall, W. S. (2004). Geometric ergodicity and perfect simulation. Electron. Commun. Prob. 9, $140-151$.

[21] Kendall, W. S. and Møller, J. (2000). Perfect simulation using dominating processes on ordered spaces, with application to locally stable point processes. Adv. Appl. Prob. 32, 844-865.

[22] Kendall, W. S., van Lieshout, M. and Baddeley, A. (1999). Quermass-interaction processes: conditions for stability. Adv. Appl. Prob. 31, 315-342.

[23] Klein, W. (1982). Potts-model formulation of continuum percolation. Phys. Rev. B 26, 2677-2678.

[24] Likos, C., Mecke, K. and Wagner, H. (1995). Statistical morphological of random interfaces in microemulsions. J. Chem. Phys. 102, 9350-9361.

[25] Mecke, K. (1994). Integralgeometrie in der Statistichen Physik (Reine Physik 25). Harri Deutsch, Frankfurt.

[26] Mecke, K. (1996). A morphological model for complex fluids. J. Phys. Condensed Matters 8, 9663-9667. 
[27] Molchanov, I. (1997). Statistics of the Boolean Model for Practitioners and Mathematicians. John Wiley, Chichester.

[28] MøLler, J. (1994). Contribution to the discussion of N. L. Hjort and H. Omre (1994): Topics in spatial statistics. Scand. J. Statist. 21, 346-349.

[29] MøLler, J. (1994). Lectures on Random Voronoi Tessellations (Lecture Notes Statist. 87). Springer, New York.

[30] MøLler, J. (1999). Markov chain Monte Carlo and spatial point processes. In Stochastic Geometry: Likelihood and Computation (Monogr. Statist. Appl. Prob. 80), ed. O. E. Barndorff-Nielsen, Chapman \& Hall/CRC, Boca Raton, FL, pp. 141-172.

[31] Møller, J. and Helisová, K. (2007). Power diagrams and interaction processes for unions of discs. Res. Rept. R-2007-15, Department of Mathematical Sciences, Aalborg University.

[32] Møller, J. ANd Helisová, K. (2008). Likelihood inference for unions of interacting discs. In preparation.

[33] Møller, J. and Waagepetersen, R. P. (1998). Markov connected component fields. Adv. Appl. Prob. 30, 1-35.

[34] Møller, J. And WaAgepetersen, R. P. (2003). Statistical Inference and Simulation for Spatial Point Processes. Chapman \& Hall/CRC, Boca Raton, FL.

[35] Nguyen, X. X. And Zessin, H. (1979). Integral and differential characterizations of Gibbs processes. Math. Nachr. 88, 105-115.

[36] Okabe, A., Boots, B., Sugihara, K. and Chiu, S. N. (2000). Spatial Tessellations. Concepts and Applications of Voronoi Diagrams, 2nd edn. John Wiley, Chichester.

[37] Preston, C. J. (1977). Spatial birth-and-death processes. Bull. Internat. Statist. Inst. 46, 371-391.

[38] Ripley, B. D. ANd Kelly, F. P. (1977). Markov point processes. J. London Math. Soc. 15, 188-192.

[39] Roberts, G. O. and Rosenthal, J. S. (1997). Geometric ergodicity and hybrid Markov chains. Electron. Commun. Prob. 2, 13-25.

[40] Ruelle, D. (1971). Existence of a phase transition in a continuous classical system. Phys. Rev. Lett. 27, 1040-1041.

[41] Stoyan, D., Kendall, W. S. and Mecke, J. (1995). Stochastic Geometry and Its Applications, 2nd edn. John Wiley, Chichester.

[42] Van Lieshout, M. N. M. (2000). Markov Point Processes and Their Applications. Imperial College Press, London.

[43] Weil, W. and Wieacker, J. (1984). Densities for stationary random sets and point processes. Adv. Appl. Prob. 16, 324-346.

[44] Weil, W. And Wieacker, J. (1988). A representation theorem for random sets. Prob. Math. Statist. 9, $147-151$.

[45] Widom, B. AND Rowlinson, J. S. (1970). a new model for the study of liquid-vapor phase transitions. J. Chem. Phys. 52, 1670-1684.

[46] WiLson, R. (1972). Introduction to Graph Theory. Oliver and Boyd, Edinburgh. 